\title{
Mitochondrial angiotensin receptors in dopaminergic neurons. Role in cell protection and aging-related vulnerability to neurodegeneration
}

\author{
Rita Valenzuela ${ }^{1,2,10}$, Maria A Costa-Besada ${ }^{1,2,10}$, Javier Iglesias-Gonzalez ${ }^{3}$, Emma Perez-Costas ${ }^{4}$, Begoña Villar-Cheda ${ }^{1,2}$, \\ Pablo Garrido-Gil ${ }^{1,2}$, Miguel Melendez-Ferro ${ }^{5}$, Ramon Soto-Otero ${ }^{6,2}$, Jose L Lanciego ${ }^{7,2}$, Daniel Henrion ${ }^{8}$, Rafael Franco ${ }^{9,2}$ and \\ Jose L Labandeira-Garcia ${ }^{*, 1,2}$
}

The renin-angiotensin system (RAS) was initially considered as a circulating humoral system controlling blood pressure, being kidney the key control organ. In addition to the 'classical' humoral RAS, a second level in RAS, local or tissular RAS, has been identified in a variety of tissues, in which local RAS play a key role in degenerative and aging-related diseases. The local brain RAS plays a major role in brain function and neurodegeneration. It is normally assumed that the effects are mediated by the cell-surfacespecific G-protein-coupled angiotensin type 1 and 2 receptors (AT1 and AT2). A combination of in vivo (rats, wild-type mice and knockout mice) and in vitro (primary mesencephalic cultures, dopaminergic neuron cell line cultures) experimental approaches (confocal microscopy, electron microscopy, laser capture microdissection, transfection of fluorescent-tagged receptors, treatments with fluorescent angiotensin, western blot, polymerase chain reaction, HPLC, mitochondrial respirometry and other functional assays) were used in the present study. We report the discovery of AT1 and AT2 receptors in brain mitochondria, particularly mitochondria of dopaminergic neurons. Activation of AT1 receptors in mitochondria regulates superoxide production, via Nox4, and increases respiration. Mitochondrial AT2 receptors are much more abundant and increase after treatment of cells with oxidative stress inducers, and produce, via nitric oxide, a decrease in mitochondrial respiration. Mitochondria from the nigral region of aged rats displayed altered expression of AT1 and AT2 receptors. AT2-mediated regulation of mitochondrial respiration represents an unrecognized primary line of defence against oxidative stress, which may be particularly important in neurons with increased levels of oxidative stress such as dopaminergic neurons. Altered expression of AT1 and AT2 receptors with aging may induce mitochondrial dysfunction, the main risk factor for neurodegeneration.

Cell Death and Disease (2016) 7, e2427; doi:10.1038/cddis.2016.327; published online 20 October 2016

The renin-angiotensin system (RAS) was initially considered as a circulating humoral system controlling blood pressure and kidney as a key control organ. The actions of angiotensin II (All), the most important effector peptide, are mediated by two main cell receptors: All type 1 and 2 (AT1 and AT2). It is generally considered that AT2 receptors exert actions directly opposed to those mediated by AT1 receptors, thus antagonizing many of the effects of the latter. ${ }^{1}$ In addition to this 'classical' humoral RAS, a second RAS, local or tissue RAS, has been identified in a variety of tissues, including the central nervous system. ${ }^{2}$ The role of RAS on brain function was initially associated with the effects of circulating RAS in areas involved in central control of blood pressure; however, it is now known that the local brain RAS is involved in different brain functions and disorders. ${ }^{3,4}$ We have previously demonstrated the presence of a local RAS in the substantia nigra pars compacta $(\mathrm{SNc})$ and striatum of rodents and primates, including humans. ${ }^{5-7}$ It has also been demonstrated that overactivation of local RAS, via AT1 receptors, exacerbates neuroinflammation, oxidative stress and dopaminergic cell

\footnotetext{
${ }^{1}$ Laboratory of Neuroanatomy and Experimental Neurology, Department of Morphological Sciences, CIMUS, University of Santiago de Compostela, Santiago de Compostela, Spain; ${ }^{2}$ Networking Research Center on Neurodegenerative Diseases (CIBERNED), Madrid, Spain; ${ }^{3}$ Healing Foundation Centre, The University of Manchester, Manchester, UK; ${ }^{4}$ Department of Pediatrics-Pediatric Nephrology, University of Alabama at Birmingham, Birmingham, AL, USA; ${ }^{5}$ Department of SurgeryPediatric, University of Alabama at Birmingham, Birmingham, AL, USA; ${ }^{6}$ Laboratory of Neurochemistry, Department of Biochemistry and Molecular Biology, Faculty of Medicine, University of Santiago de Compostela, Santiago de Compostela, Spain; ${ }^{7}$ Neuroscience Department, Center for Applied Medical Research (CIMA, IdiSNA ), University of Navarra, Pamplona, Spain; ${ }^{8}$ MITOVASC Institute, INSERM U1083, CNRS UMR6214, University of Angers, Angers, France and ${ }^{9}$ Laboratory of Molecular Neurobiology, Department of Biochemistry and Molecular Biology, Faculty of Biology, University of Barcelona, Barcelona, Spain

*Corresponding author: JL Labandeira-Garcia, Department of Morphological Sciences, Laboratory of Neuroanatomy and Experimental Neurology, Faculty of Medicine, University of Santiago de Compostela, Santiago de Compostela 15782, Spain. Tel: +34 881812 223; Fax: +34 881812 378; E-mail: joseluis.labandeira@usc.es

${ }^{10}$ These authors contributed equally to this work.

Abbreviations: All, angiotensin II; AT1, angiotensin type 1 receptors; AT2, angiotensin type 2 receptors; BCA, bicinchoninic acid; COX, cytochrome $c$ oxidase; DPI, diphenyleneidonium; E, maximum respiratory rate; ETC, electron transport chain; ETS, electron transport system; HDAC2, histone deacetylase 2; LCM, laser captured microdissection; LDH, lactate dehydrogenase; mitoKATP, ATP-sensitive potassium channels; MTDR, Mitotracker Deep Red; NAC, N-acetyl-cysteine; NO, nitric oxide; NOS, nitric oxide synthase; Nox4, NADPH oxidase 4; $\mathrm{O}_{2}^{-}$, superoxide; $\mathrm{P}$, oxidative phosphorylation; PD, Parkinson's disease; RAS, renin-angiotensin system; ROS, reactive oxygen species; RT-PCR, reverse trascription polymerase chain reaction; SN, substantia nigra; SNc, substantia nigra pars compacta; TH, tyrosine hydroxylase; VDAC, voltage-dependent anion channel; VM, ventral mesencephalon; WB, western blot

Received 27.6.16; revised 14.9.16; accepted 16.9.16; Edited by A Verkhratsky
} 
death, all of which are inhibited by treatment with AT1 receptor antagonists. $^{8,9}$

More recently, a third-level of RAS (i.e. intracellular/ intracrine) has been suggested in peripheral tissues. ${ }^{10,11}$ The system may be activated by All internalized using AT1 receptors or by intracellularly synthesized All. ${ }^{12}$ Immunohistochemical studies suggest an apparent intracellular localization of several RAS components in the SNc of mammals, including primates and humans. ${ }^{5,13}$ Mitochondrial dysfunction plays a major role in several neurodegenerative disorders, particularly in the degeneration of dopaminergic neurons in Parkinson's disease (PD). In the present study, we have discovered AT1 and AT2 receptors in brain mitochondria and investigated their role in controlling mitochondrial events. The experiments were carried out in rats, in AT1 and AT2 receptor knockout mice, in primary cultures of the nigral region and in the dopaminergic neuron cell line MES 23.5. We carried out functional studies with isolated mitochondria to exclude any possible indirect effects caused by non-mitochondrial AT1 and AT2 receptors, and showed that angiotensin receptors control key mitochondrial events.

\section{Results}

Localization of angiotensin receptors in mitochondria of dopaminergic neurons in cell cultures and rat substantia nigra. The localization of AT1 and AT2 receptors in dopaminergic neurons has been shown by immunohistochemistry in previous studies. In the present study, this was confirmed by laser captured microdissection (LCM) of dopaminergic neurons in the rat substantia nigra (SN) and reverse trascription polymerase chain reaction (RT-PCR). RTPCR analysis revealed expression of detectable mRNA levels of $\mathrm{TH}$, angiotensinogen and AT1 and AT2 receptors in isolated nigral dopaminergic neurons (Figure 1a).

We used triple immunofluorescence and confocal microscopy to investigate whether mitochondria of dopaminergic neurons expressed AT1 and AT2 receptors. Series of confocal images were obtained every $0.7 \mu \mathrm{m}$ in the Z-axis level by a sequential scan method. Dopaminergic neurons were identified by their tyrosine hydroxylase (TH)-immunoreactivity, and mitochondria were labeled with the specific probe Mitotracker Deep Red (MTDR), which revealed the characteristic pattern of the mitochondrial network. The specificity of the antibodies (see Materials and Methods) was confirmed by western blot (WB) analysis of lysates from HEK293 cells transfected with recombinant AT1 or AT2 containing a C-terminal DDK epitope tag (DYKDDDDK) fused (Origene). A predominant immunoreactive band was observed in positive transfected lysates but not in negative controls, which consisted of empty vector transfected lysates (Figure 1b).

In primary cultures (Figure 1c) and the MES 23.5 cell line of dopaminergic (i.e. TH-positive) neurons (Figure 1d), dopaminergic neurons showed intense inmunoreactivity to $\mathrm{TH}$ antibody. Labeling for AT1 receptors was intense at the periphery of the cells, suggesting the presence of receptors at the cell surface, and also within the neurons, at cytoplasmic and nuclear levels. Colocalization of AT1 with the specific probe MTDR revealed that many of the mitochondria expressed AT1 receptors, although some mitochondria showed weak or unclear AT1 immunolabeling. On the contrary, AT2 receptor labeling was particularly intense in the cytoplasm, while weaker labeling was observed at the cell surface and in the nucleus. At the cytoplasmic level, AT2 labeling colocalized with MTDR and the pattern of distribution was similar to that of mitochondrial labeling, which suggests a strong presence of this receptor in mitochondria of dopaminergic neurons.

Electron microscopy samples were obtained from the densely packed dopaminergic cell clusters of the rat substantia nigra compacta. Labeling for both AT1 and AT2 receptors was observed in the neuronal processes (Figure 1e) and in the cytoplasm of dopaminergic neurons (Figure 1f), associated with different organelles and cytoplasmic structures. More specifically, labeling was frequently observed in mitochondria, mainly located in the outer membrane (Figures 1e and f) although in some cases labeling was also observed in the cristae (Figure 1f, left). Labeling for both receptors was also observed in the rough endoplasmic reticulum, and in clusters of free ribosomes, which were often located in close vicinity to labeled mitochondria (Figures 1e and f).

Presence of fluorescent-tagged angiotensin receptors and fluorescent All in mitochondria. Twenty-four hours after transient transfection of the dopaminergic neuron cell line MES 23.5, AT1 and AT2 receptors labeled with fluorescent proteins (yellow fluorescent protein, YFP; enhanced green fluorescent protein, EGFP) were located both at the plasma membrane and intracellularly, and they colocalized with the mitochondrial marker MDTR (Figures 2a-f). In a second series of experiments, cultures of the dopaminergic neuron cell line MES 23.5 were treated with Alexa Fluor 488-conjugated All to investigate the localization of All within the mitochondria. The fluorescent All was internalized and colocalized with mitochondria, which was evident after $30 \mathrm{~min}$, and persisted for $8 \mathrm{~h}$ after treatment (Figures 2g-i).

Interestingly, enhanced intracellular oxidative stress (i.e. after treatment of cells with very low doses of $\mathrm{MPP}^{+}$) altered the distribution of AT2-YFP labeling. In particular, after labeling mitochondria with a mitotracker probe, AT2-YFP was more abundant at the mitochondrial level than in untreated cells. However, treatment of cells with the antioxidant $N$-acetylcysteine (NAC) induced increased mitochondrial AT1-EGFP fluorescence relative to control cells. These effects were confirmed by WB in samples from isolated mitochondria (see below).

Presence of angiotensin receptors and All in isolated mitochondria. Mitochondria were isolated from the nigral region of rat in the ventral mesencephalon (VM) by separating organelles by ultracentrifugation in a preformed Percoll gradient. ${ }^{14}$ The quality of the sample was demonstrated by the absence of different specific cell compartment markers, such as histone deacetylase 2 (HDAC2) and $a$-tubulin, which are markers for the nuclear and the cytosol fractions, respectively. The mitochondrial fraction was confirmed with the mitochondrial marker voltage-dependent anion channel (VDAC). The activity of lactate dehydrogenase 
a
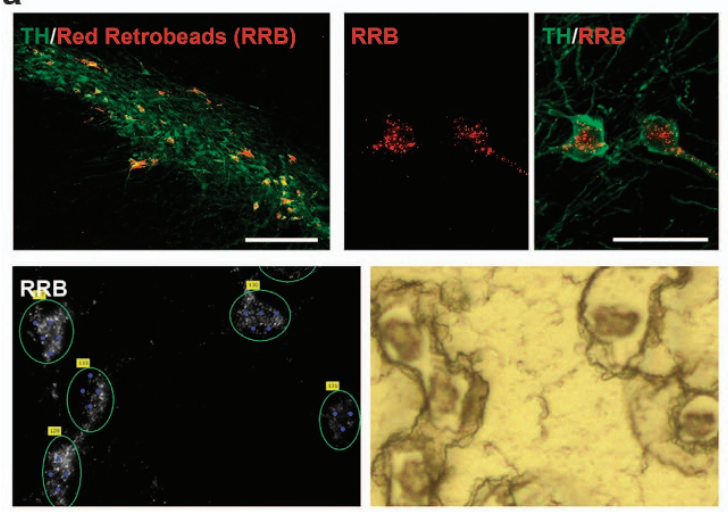

C
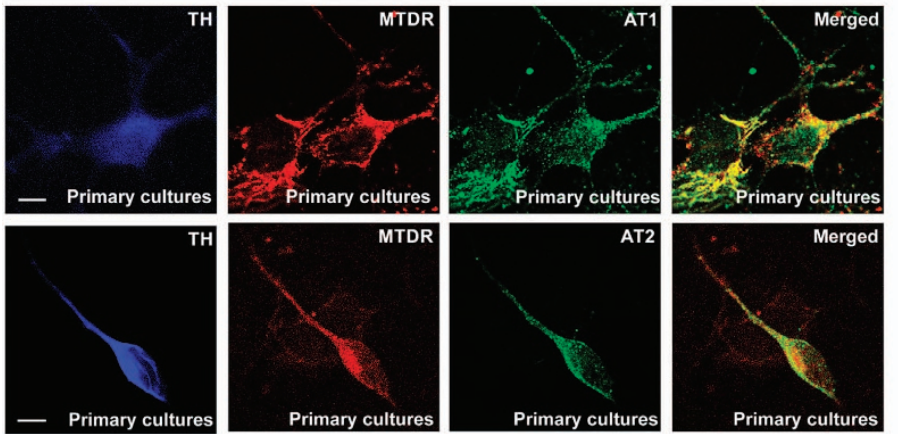

b

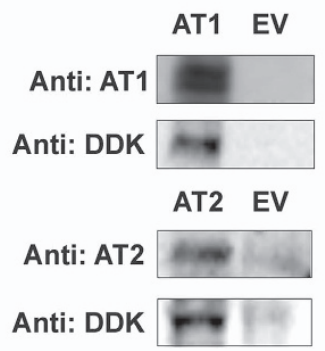

d

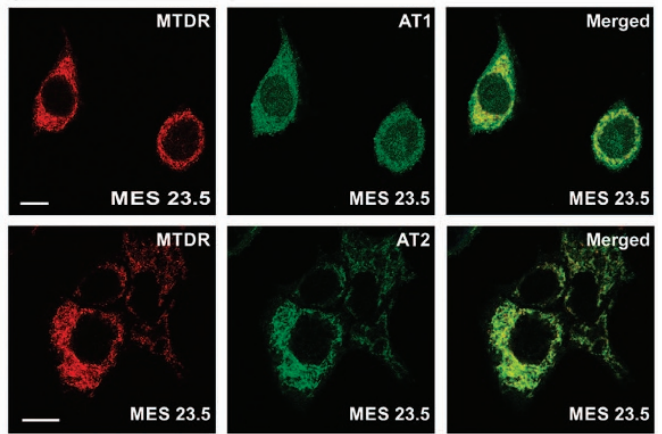

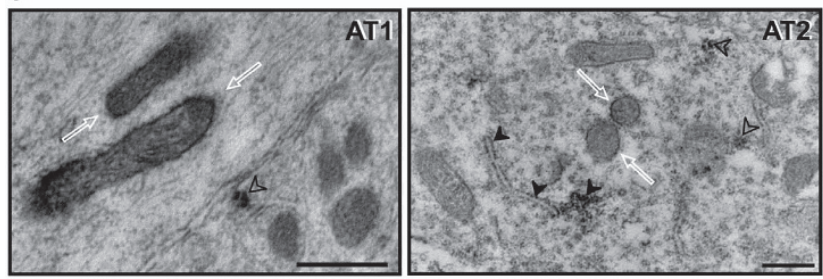

f

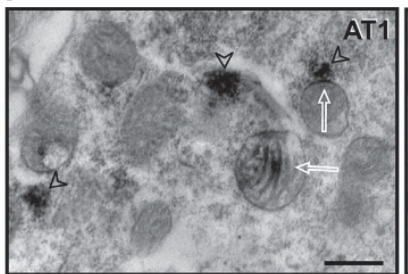

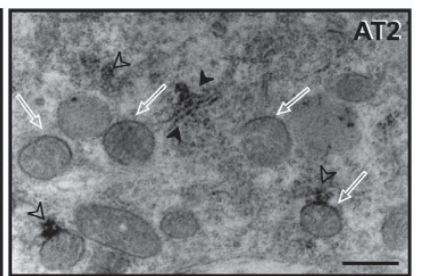

Figure 1 Specificity of angiotensin receptor antibodies and localization of angiotensin receptors in mitochondria of dopaminergic neurons. (a) The expression of major RAS components in dopaminergic neurons was confirmed by RT-PCR and laser microdissection of dopaminergic neurons retrogradly labeled by intrastriatal injection of fluorescent red retrobeads (RRB). SN section showing labeled dopaminergic neurons before and after laser microdissection for RT-PCR. Expression of TH, AGT, AT1, AT2 and $\beta$-actin mRNA in laser-microdissected dopaminergic neurons (right) and homogenates of SN used as a positive control (left) are also shown. (b) Western blot densitometric bands corresponding to $10 \mu \mathrm{g}$ of AT1 or AT2 receptor overexpression lysate containing a C-terminal DDK epitope tag (DYKDDDDK) fused (left), and $10 \mu \mathrm{g}$ of empty vector transfected control cell lysate HEK293 (EV, right). A band of $45 \mathrm{kDa}$ was detected with the AT1 antibody, while a band of $50 \mathrm{kDa}$ was detected with the AT2 antibody. A monoclonal antibody against DDK detected the corresponding band in the protein lysates. Colocalization of mitochondria and AT1 and AT2 receptors in primary cultures of the nigral region (c) and neurons from the MES 23.5 dopaminergic cell line (d). Electron microscopy of AT1 and AT2 labeling in a neuronal process (e) and cytoplasm (f) of a dopaminergic neuron. Immunolabeling for AT1 was observed in the outer membrane and cristae (white arrows) of mitochondria. In addition, strong AT1 labeling was also seen in clusters of free ribosomes (empty arrowheads). Immunolabeling for AT2 was present in mitochondrial membranes (white arrows), in the rough endoplasmic reticulum (black arrowheads) and in small clusters of free ribosomes, some of which were in close proximity to labeled mitochondria (empty arrowheads). AGT, angiotensinogen; DA, dopaminergic; MTDR, MitoTracker Deep Red; SN, substantia nigra; TH, tyrosine hydroxylase. Scale bars: (a) 50 and $200 \mu \mathrm{m}$ (SN section); (c) $5 \mu \mathrm{m}$; (d) $10 \mu \mathrm{m}$; (e and f) $500 \mathrm{~nm}$

(LDH), which is a cytosolic enzyme predominantly associated with contaminating synaptosomes, was measured relative to that of the whole homogenate, and only residual nonsignificant activity was observed in the mitochondrial fraction (Figures $3 a-d)$. We observed the presence of the main angiotensin receptor types (i.e. AT1 and AT2) in the mitochondrial fraction. There was a clear difference in the abundance of each receptor subtype; while AT1 appeared less abundant in mitochondria than in total cell homogenate, AT2 expression was higher than in the total homogenate
(Figures $3 a-c)$. Furthermore, we have confirmed the presence of All peptide in isolated mitochondria using HPLC purified samples $(0.0381 \pm 0.00618 \mathrm{pg} / \mathrm{ml})$.

Effects of oxidative stress levels and aging on the expression of mitochondrial angiotensin receptors. After transient transfection of the dopaminergic neuron cell line MES 23.5, AT2-YFP was more abundant at the mitochondrial level after treatment of cells with inducers of oxidative stress such as very low doses of $\mathrm{MPP}^{+}$; relative 

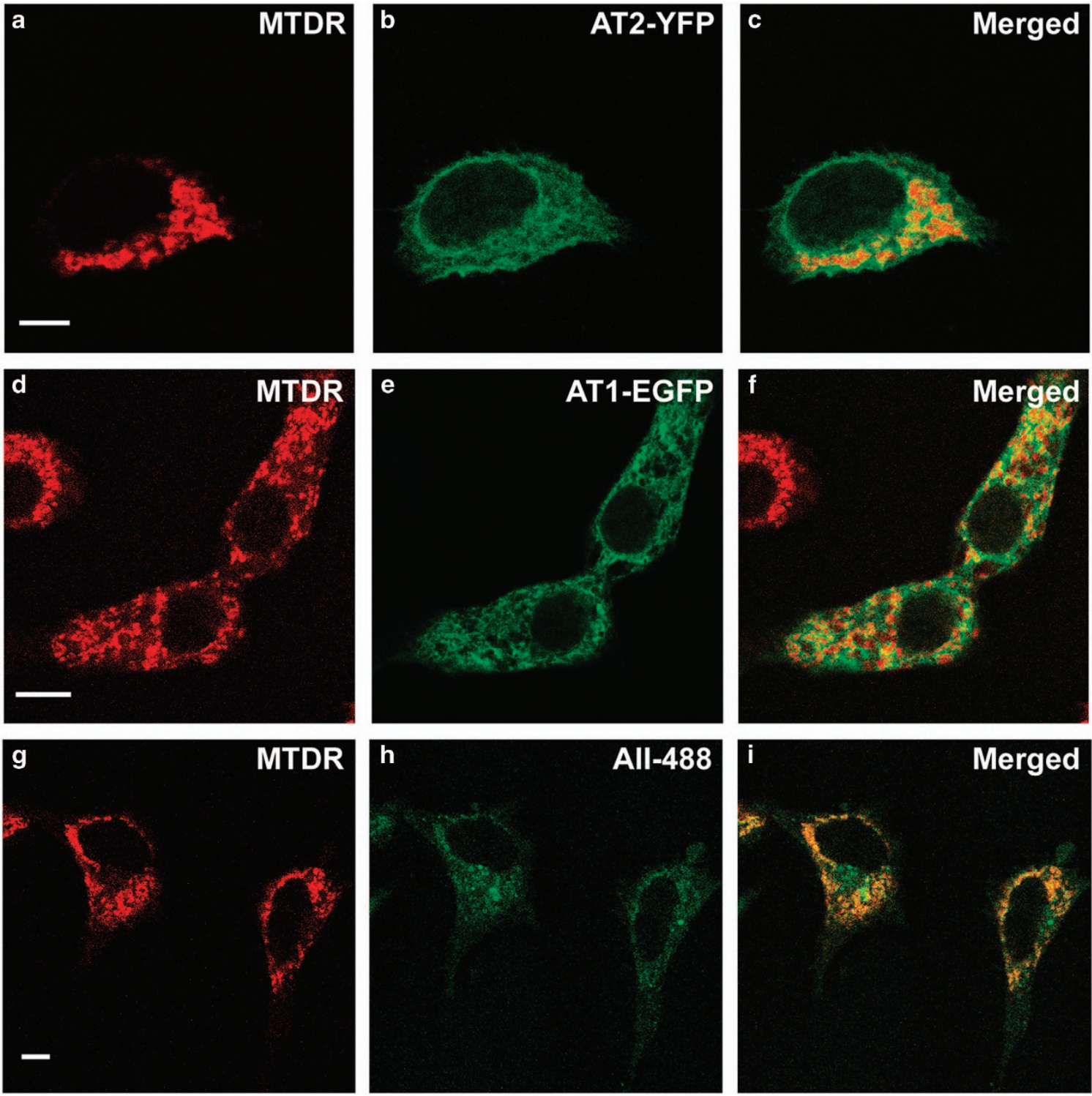

Figure 2 Presence of fluorescence-tagged angiotensin receptors and fluorescent angiotensin II in mitochondria. Colocalization (c, $f$ and $\mathrm{i})$ of the fluorescent mitochondrial marker MTDR (a, d and $\mathbf{g}$ ) with AT2-YFP (b), AT1-EGFP $(\mathbf{e})$ or All-488 (h). Fluorescent All-488 colocalized with mitochondria $8 \mathrm{~h}$ after treatment of cultures $(\mathbf{g}-\mathbf{i})$. Cells transfected with AT1-EGFP required simultaneous treatment with the AT1 receptor antagonist losartan, in order to minimize AT1-induced superoxide toxicity and cell death. AT2YFP, angiotensin receptor type 2 tagged to yellow fluorescent protein; AT1-EGFP, angiotensin receptor type 1 tagged to enhanced green fluorescent protein; All-488, Alexa Fluor 488-fluorescent angiotensin II; MTDR, MitoTracker Deep Red. Scale bar: $5 \mu \mathrm{m}$

density of the protein bands normalized to VDAC were $94.9 \pm 13.0$ for untreated controls and $248.8 \pm 44.3$ for cells treated with $\mathrm{MPP}^{+}(P<0.05)$. Treatment of cells with the antioxidant NAC led to higher mitochondrial levels of AT1EGFP than in mitochondria from untreated dopaminergic cells (Control: $103.1 \pm 5.0 ; \quad$ NAC: $130.6 \pm 7.8 ; \quad P<0.05$; Figures $3 e$ and $\mathrm{f}$ ).

RT-PCR analysis of dopaminergic neurons isolated from rat SN using LCM revealed that aging induces a significant increase (45\%) in AT1 receptor mRNA expression. In contrast, the levels of AT2 receptor mRNA in dopaminergic neurons were significantly lower ( $83 \%$ decrease) in aged rats than in young rats (Figures $3 \mathrm{~g}$ and $\mathrm{h}$ ). In isolated mitochondria, the expression of mitochondrial AT1 receptors detected by WB increased in aged rats, while expression of mitochondrial AT2 was lower in aged rats than in young rats (Figure 3i). Furthermore, using the same isolation procedure, there was a substantial difference in total protein concentration between mitochondria isolated from young and aged rats. Mitochondria isolated from aged rats contained almost half the amount of protein concentration than those from young rats. This is not surprising since the number and mass of mitochondria is known to decrease with age, which alters the quantification of protein between both groups of animals. Therefore, to obtain a 
more accurate estimation of mitochondrial content, we used cytochrome $c$ oxidase (COX) activity to normalize the data obtained from AT1 and AT2 receptor expression determined by WB (Figure $3 \mathrm{i}){ }^{15}$
Effect of mitochondrial angiotensin receptors on mitochondrial respiration. Bioenergetic studies were carried out using mitochondria isolated from the nigral region of rat, and confirmed with mitochondria isolated from the whole brain. a

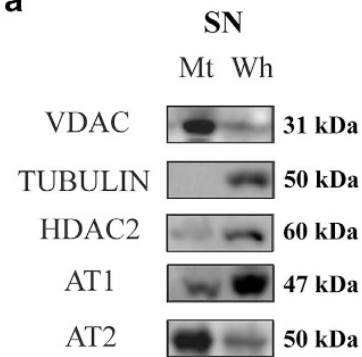

b

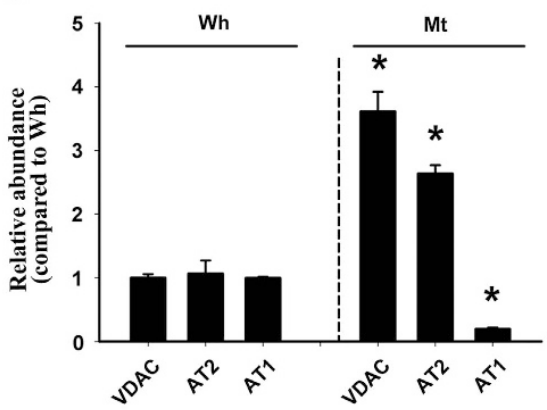

C

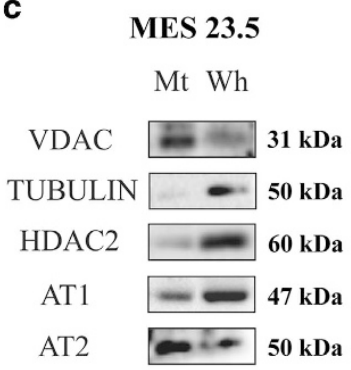

d

\begin{tabular}{|c|c|c|c|}
\hline & \multicolumn{2}{|c|}{ LDH specific activitv } & \multirow[t]{2}{*}{ Relative activitv } \\
\hline & Mt & Wh & \\
\hline SN & $0.00981 \pm 0.00212$ & $0.0546 \pm 0.00418$ & 0.179 \\
\hline MES 23.5 & $0.0591 \pm 0.00544$ & $0.187 \pm 0.0151$ & 0.316 \\
\hline
\end{tabular}

\section{AT2 (MES 23.5)}

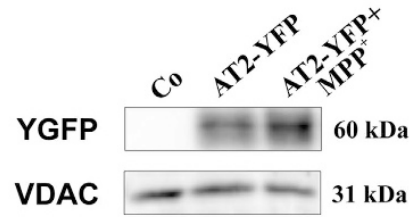

g
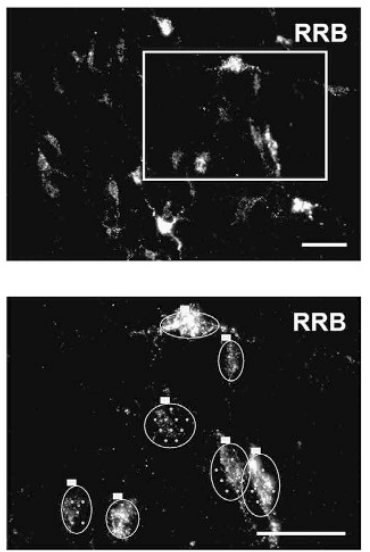

AT1 (MES 23.5)

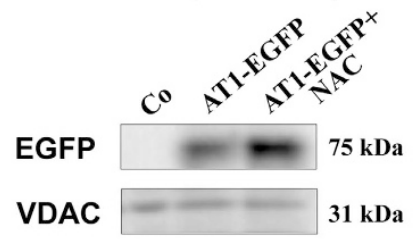

h

DA NEURONS
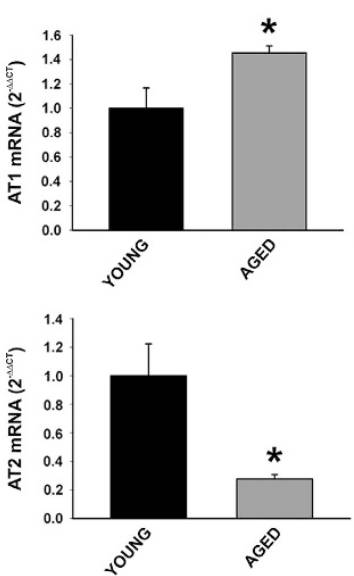

f

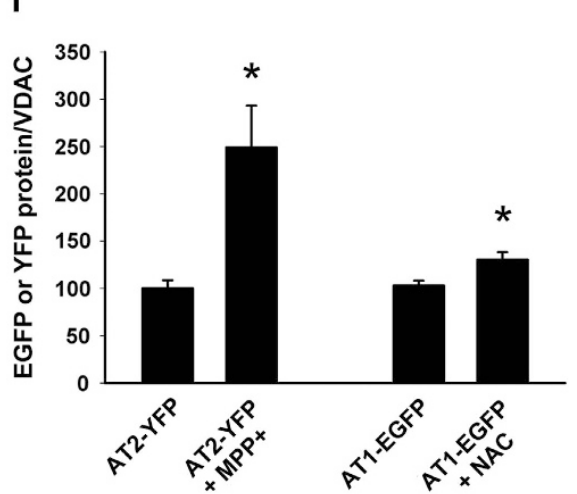

i
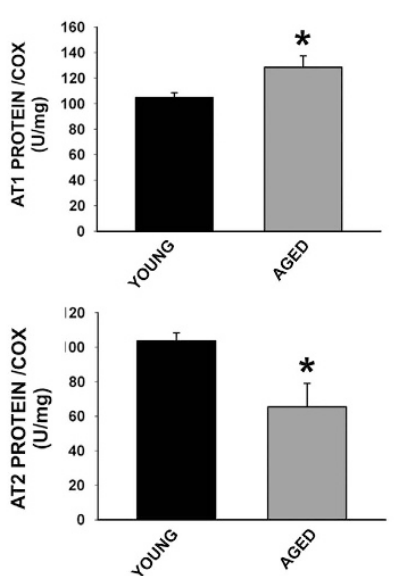

Figure 3 AT1 and AT2 receptors in isolated mitochondria (IM) from ventral mesencephalon and the dopaminergic neuron cell line MES 23.5. Effect of oxidative stress and aging. (a-c) Western blot (WB) of whole homogenate (Wh; $n=4)$ and pure IM (Mt; $n=4)$ from the nigral region (a) and MES 23.5 cells (c) showing different compartment markers used to assess the purity of the IM: voltage-dependent anion channel (VDAC) as a mitochondrial marker, tubulin as a cytosol marker and histone deacetylase 2 (HDAC2) as a nuclear marker. Note the higher expression of mitochondrial AT2 compared with AT1 receptors (b). (d)The levels of lactate dehydrogenase specific activity (LDH; micromol of substrate/min/mg; a marker for cytosolic and synaptosomal contamination) of the nigral region and MES 23.5 pure IM (Mt; $n=6)$ were negligible relative to those in Wh. (e and f) WB of IM from the MES 23.5 cells transfected with AT2-YFP (yellow fluorescent protein; $n=4$ ) or AT1-EGFP (enhanced green fluorescent protein; $n=3$ ) showing the presence of fluorescent-tagged angiotensin receptors in IM in comparison with non-transfected cells ( $\mathrm{Co}$, control); $24 \mathrm{~h}$ treatment of cells, low doses of MPP $(n=4)$ or $\mathrm{N}$-acetyl-L-cysteine (NAC; $n=3$ ) induced increased expression of mitochondrial AT2 and AT1 receptors, respectively (AT2-YFP+MPP+; AT1-EGFP+NAC). (g-i) In young ( $n=3)$ and aged $(n=4)$ rats, the expression of AT1 and AT2 receptors was analyzed by RT-PCR in nigral dopaminergic neurons labeled with red retrobeads (RRB) and isolated by laser microdissection $(\mathbf{g}-\mathbf{h})$, and by WB of IM from the nigral region of young $(n=5-8)$ and aged rats $(n=5-6)$ (i). Aging induced a significant increase in AT1 expression and a significant decrease in AT2 expression in both dopaminergic neurons and in IM. The results were normalized to the values of young animals. Data are means $\pm S$.E.M. ${ }^{*} P<0.05$ relative to the corresponding controls (Student's t-test). Scale bars: $50 \mu \mathrm{m}$ 
Isolated mitochondria were treated with All in the presence of antagonists of either AT1 (losartan) or AT2 (PD123,319) receptors. Our results showed that activation of mitochondrial angiotensin receptors produced opposite effects on respiratory function. Activation of mitochondrial AT1 receptors with All (i.e. All+ PD123,319) induced an increase in both
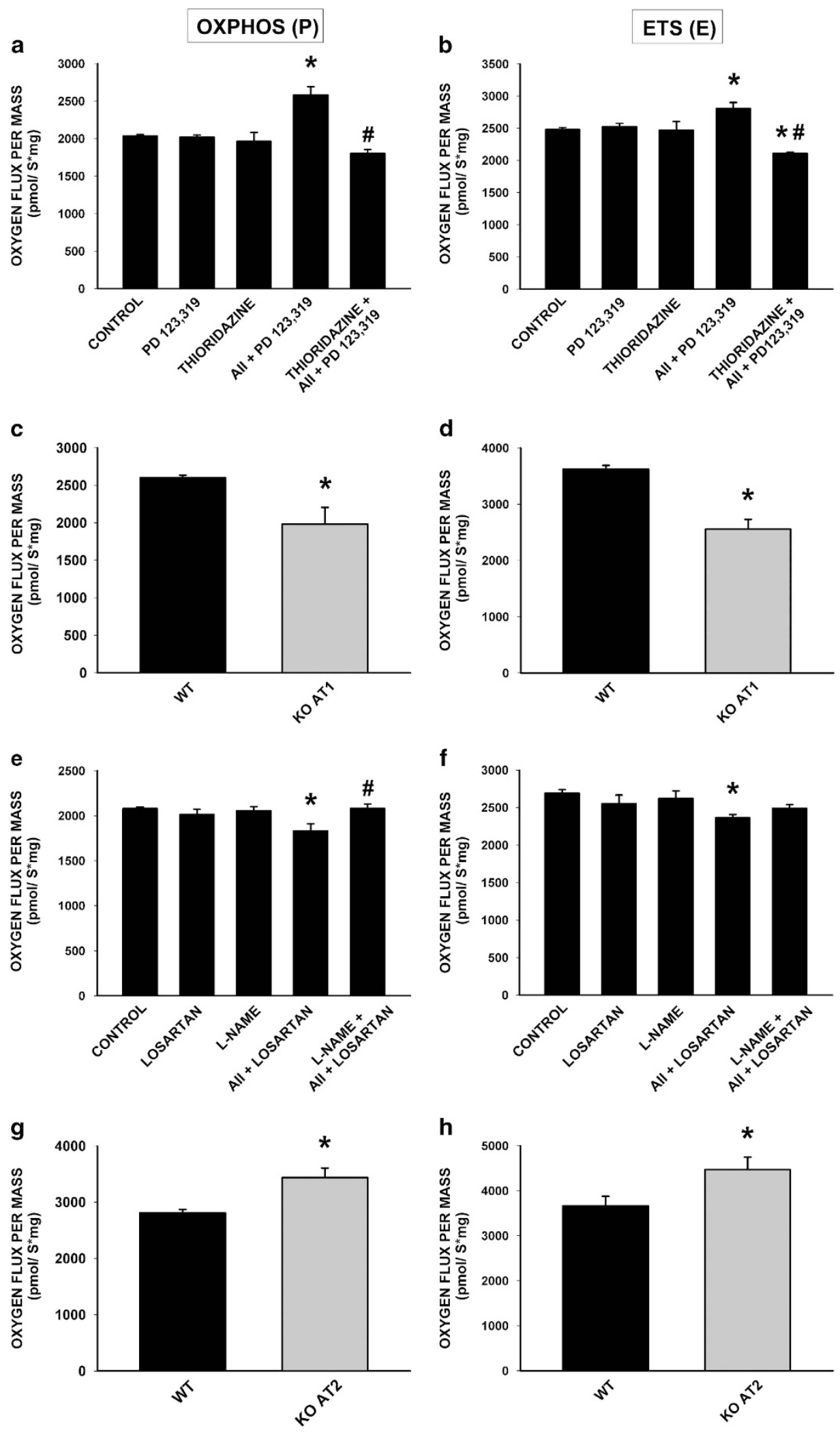
oxidative phosphorylation $(\mathrm{P})$ and maximum respiratory rate (E) (Figures $4 a$ and b). This was consistent with that observed in mice lacking AT1 receptors, which showed a decrease in respiratory activity compared with wild-type mice (Figures $4 \mathrm{c}$ and $\mathrm{d}$ ). Activation of mitochondrial AT2 receptors by All (i.e. All+losartan) produced a significant decrease in activated respiration (OXPHOS or $\mathrm{P}$ ) and maximum respiration rate (maximum electron transport system, ETS or E) associated with complex I (Figures $4 e$ and f). This is consistent with the results in knockout mice for AT2 receptors, which showed higher mitochondrial respiration rates compared with wild-type littermate controls (Figures $4 g$ and $h$ ).

Interestingly, the increase in respiratory capacity induced by activation of mitochondrial AT1 receptor was blocked by preincubation of isolated mitochondria with the NOX4 inhibitor thioridazine, which revealed the major role of NOX4 in the induction of superoxide by mitochondrial AT1 receptor activation (Figures $4 a$ and $b$ ). The decrease in respiratory capacity induced by activation of mitochondrial AT2 receptors was inhibited by pre-treatment of isolated mitochondria with the nitric oxide synthase (NOS) inhibitor, $N^{\omega}$-nitro-L-arginine methyl ester hydrochloride (L-NAME), which revealed the major role of nitric oxide (NO) in this effect (Figures $4 e$ and f).

Effect of mitochondrial angiotensin receptors on mitochondrial transmembrane potential, nitric oxide and superoxide production. Role of mitochondrial NOS and mitochondrial NADPH oxidase 4. Isolated mitochondria were incubated with the AT1 receptor antagonist losartan or the AT2 receptor antagonist PD123,319, and then incubated with All. Mitochondria were energized through complex I by adding pyruvate and malate. Activation of AT1 or AT2 receptors did not induce any significant change in mitochondrial membrane potential, indicating that the bioenergetic properties of the mitochondria were not affected (Figure 5a). As control, the potassium ionophore valinomycin was added to the sample at a final concentration of $0.5 \mu \mathrm{g} / \mathrm{ml}$, which led to loss of approximately $40 \%$ of transmembrane potential relative to non-treated mitochondria.

In isolated mitochondria, activation of AT2 receptors (i.e. treatment with All and the AT1 antagonist losartan) induced an increase in levels of NO that was inhibited by pre-treatment with the NOS inhibitor L-NAME (Figure 5b). This is consistent with the mitochondrial respiration data, which showed that AT2-dependent respiratory inhibition was prevented by treatment of isolated mitochondria with L-NAME, confirming the link between mitochondrial AT2 receptors and NO production, and the mitochondrial respiratory function.
Mitochondrial superoxide $\left(\mathrm{O}_{2}^{-}\right)$production was measured by lucigenin-enhanced chemiluminescence. In isolated mitochondria, activation of AT1 receptors with All (i.e. treatment with All and the AT2 receptor antagonist PD123,319) resulted in increased levels of superoxide. The major sources of reactive oxygen species (ROS) in the cell are NADPH oxidase (Nox) proteins and the mitochondrial electron transport chain (ETC). Nox4 is the main intracellular form of the Nox protein family in several types of cells. ${ }^{16,17}$ In the present study, the presence of Nox4 in rat brain isolated mitochondria was demonstrated using a specific rabbit monoclonal antibody, which showed a $60 \mathrm{kDa}$ band that increased with the amount of mitochondrial sample loaded in the SDS gel (Figure $5 \mathrm{c}$ ). To clarify the role of Nox4 on superoxide production, we tested the effect of the Nox4 inhibitor thioridazine. ${ }^{18}$ Treatment of isolated mitochondria with All and the AT2 blocker PD123,319 (i.e. activation of AT1 receptors) and simultaneous treatment with thioridazine led to the inhibition of total superoxide to about a $40 \%$ of untreated controls. The remaining superoxide was probably due to mitochondrial electron leakage at the ETC or other sources (Figure 5d). This is consistent with the findings on respiratory function that showed that pre-treatment of isolated mitochondria with the Nox4 inhibitor thioridazine blocked the AT1-induced increase in oxygen consumption (Figures $4 \mathrm{a}$ and $\mathrm{b}$ ). The role of Nox4 in All-induced superoxide production was confirmed with a second Nox4 inhibitor (diphenyleneidonium, DPI). As observed after treatment with thioridazine (Figure 5d), simultaneous treatment with DPI (All +PD123,319+DPI) led to inhibition of total superoxide to about $40-50 \%$ of untreated controls (data not shown; see Materials and Methods, Superoxide production assay).

\section{Discussion}

Mitochondria play a major role in aging and aging-related neurodegenerative disorders such as degeneration of dopaminergic neurons and PD. ${ }^{19}$ The SN of PD patients shows alteration of mitochondrial NADPH dehydrogenase (complex I) activity, and complex I inhibitors such as MPTP, rotenone and other pesticides cause neurological changes similar to those observed in PD. ${ }^{20,21}$ Previous immunohistochemical findings from our group suggest the possible existence of an intracellular or intracrine RAS in dopaminergic neurons of some mammalian species, including humans. ${ }^{5,13}$ The present study demonstrates the presence of All and the main All receptors (i.e. AT1 and AT2) in the mitochondria of dopaminergic neurons by immunofluorescence and electron microscopy. This was also observed using fluorescent-tagged angiotensin receptors and fluorescent angiotensin. The

\footnotetext{
Figure 4 Effect of mitochondrial angiotensin receptors on mitochondrial respiration. (a and $\mathbf{b}$ ) Activation of mitochondrial AT1 receptors with All (i.e. All+ PD123,319) induces an increase in both oxidative phosphorylation $(P)$ and maximum respiratory rate $(E)$, which was inhibited by pre-treatment of isolated mitochondria with the NOX4 inhibitor thioridazine (n=3-8). (c and d) Knockout mice for AT1 receptors (KO AT1; $n=5$ ) show lower mitochondrial respiration rates compared with wild-type littermate controls (WT). (e and f) Activation of mitochondrial AT2 receptors by All (i.e. All+losartan) produces a significant decrease in activated respiration (OXPHOS, P) and maximum respiration rate (maximum electron transport system, ETS, E ) associated with complex I, which was blocked by pre-incubation of isolated mitochondria with the nitric oxide synthase (NOS) inhibitor, $N^{w}$-nitro-L-arginine methyl ester hydrochloride (L-NAME; $n=5-8$ ). (g and $\mathbf{h}$ ) Mice lacking AT2 receptors (KO AT2; $n=5$ ) show an increased respiratory activity compared with wild-type mice (WT). Data are mean \pm S.E.M. ${ }^{*} P<0.05$ compared with control. ${ }^{\#} P<0.05$ compared with the group treated with All. One-way analysis of variance (ANOVA) and Bonferroni post hoc test (a, $\mathbf{b}, \mathbf{e}, \mathbf{f})$ and Student's t-test $(\mathbf{c}, \mathbf{d}, \mathbf{g}, \mathbf{h})$
} 


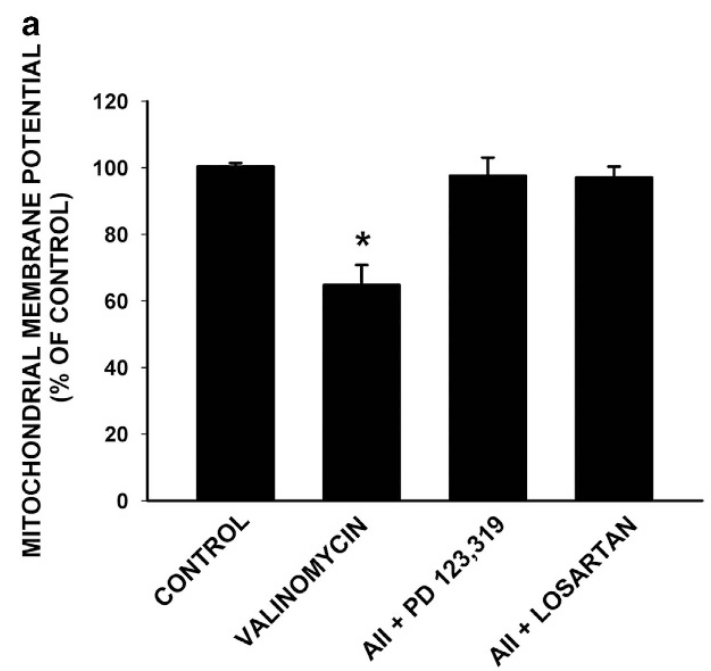

b

c

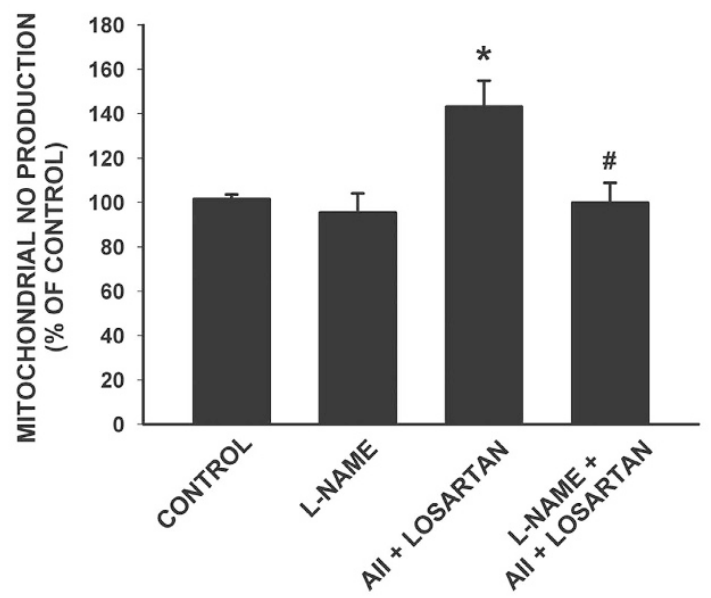

d
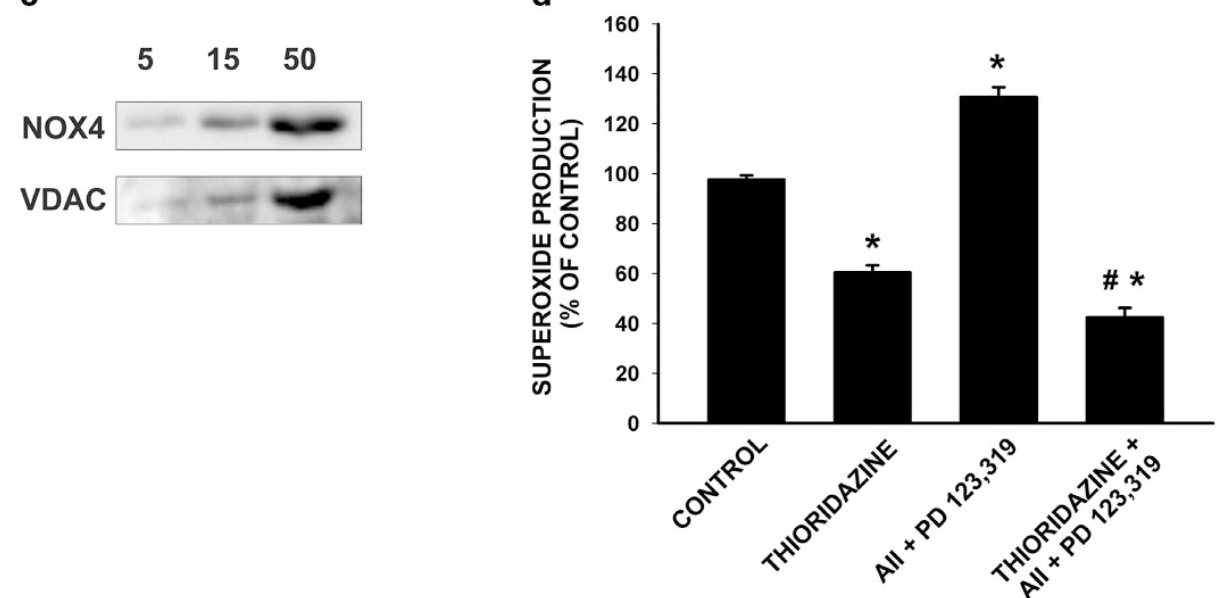

Figure 5 Effect of mitochondrial angiotensin receptors on mitochondrial transmembrane potential, nitric oxide and superoxide production. (a) In isolated mitochondria, activation of AT1 and AT2 receptors (All+ the AT2 blocker PD123,319, and All+ the AT1 blocker losartan, respectively) did not induce any significant change in mitochondrial membrane potential maintenance; however, the potassium ionophore valinomycin led to loss of approximately $40 \%$ of transmembrane potential relative to non-treated mitochondria $(n=5)$. (b) Activation of AT2 receptors (All+ the AT1 blocker losartan) induced an increase in levels of NO that was inhibited by pre-treatment with the NOS inhibitor L-NAME $(n=5-8)$. (c) The presence of Nox4 in isolated mitochondria was also shown by western blot assay with a rabbit monoclonal anti-Nox 4 antibody, which detected a band of $60 \mathrm{kDa}$, and an antibody against the mitochondrial marker VDAC. The Nox4 signal increased with mitochondrial content $(5,10,50 \mu \mathrm{g}$ of mitochondrial sample loaded in the acrylamide gel). (d) In isolated mitochondria, activation of AT1 receptors with All (All+the AT2 receptor antagonist PD123,319) resulted in increased levels of superoxide, and simultaneous treatment with the NOX4 inhibitor thioridazine led to the inhibition of total superoxide to about a $40 \%$ of untreated controls $(n=4-8)$. Data are mean \pm S.E.M. ${ }^{*} P<0.05$ compared with control. ${ }^{\#} P<0.05$ compared with the group treated with All (one-way analysis of variance (ANOVA) and Bonferroni post hoc test). L-NAME, L-arginine methyl ester hydrochloride; NOS, nitric oxide synthase

results were confirmed in mitochondrial fractions isolated from the nigral region of rat and the dopaminergic neuron cell line MES 23.5. Our results show a differential distribution of both types of All receptors; while AT2 were mainly cytoplasmic and colocalized with mitochondrial labeling, AT1 had more superficial and nuclear distribution, although we also found AT1 receptors in mitochondria. Furthermore, there was a clear difference in the expression of AT1 and AT2 in the mitochondrial fraction, with AT2 being much more abundant than AT1.

The present findings show that mitochondrial AT1 and AT2 receptor activation produced opposite effects on respiratory rates, which is consistent with that observed in AT1 and AT2 cell surface receptors. Activation of AT2 receptors induced a moderate but significant decrease in active and maximal respiration (OXPHOS and ETS). The effect was confirmed in mitochondria isolated from mice deficient in AT2 receptors, which showed an increase in respiratory rates. The possible mechanisms involved in these effects were then investigated. We found that mitochondrial AT2 receptor activation induced an increase in mitochondrial NO levels, which was blocked by inhibition of mitochondrial NOS activity by L-NAME, which also blocked the reduction in respiratory activity induced by AT2 activation, indicating that NO mediates this process and may act as a respiratory modulator. Initial observations considered NO as proapoptotic; however, it is now known that this occurs only at high concentrations of $\mathrm{NO}$, and that physiological levels of NO are antiapoptotic. ${ }^{22}$ At physiological concentrations, NO competes with oxygen by the active site of cytochrome 
oxidase. Short- term inhibition of complex IV by low nonlethal levels of NO inhibits respiration and initiates a protective action to maintain the membrane potential, which results in protection of the cell against further damage and prevents apoptosis. $^{23,24}$ AT2-mediated regulation of mitochondrial respiration may represent an unrecognized primary line of defence against oxidative stress and stress-associated damage. It is known that levels of oxidative stress are increased in dopaminergic neurons, probably related to dopamine metabolism, and the above-mentioned defence mechanism may be particularly important against dopaminergic degeneration and PD.

Mitochondrial AT1 activation produced an increase in oxygen consumption, and increased generation of superoxide via mitochondrial Nox4. This effect was confirmed in mitochondria isolated from mice deficient in AT1 receptors, which showed a decrease in respiratory rates, and is also consistent with previous observations for cell surface AT1 receptors and membrane NADPH oxidase. Our previous studies in animal models of PD showed that All, via cell surface AT1 receptors, increases oxidative stress through activation of membranebound NADPH oxidase..$^{7,9,25}$ Within the mitochondria, the primary site of ROS generation is the ETC, as leakage of electrons at complexes I and III leads to a partial reduction of oxygen to form $\mathrm{O}_{2}^{-}$. In addition, the Nox4 isoform, a member of the NADPH oxidase family, has been localized in intracellular membranes of cardiomyocytes, and renal cells. ${ }^{16,26,27}$ In the present study, we demonstrated expression of Nox4 in mitochondria isolated from the nigral region, which is consistent with the results of previous studies that showed

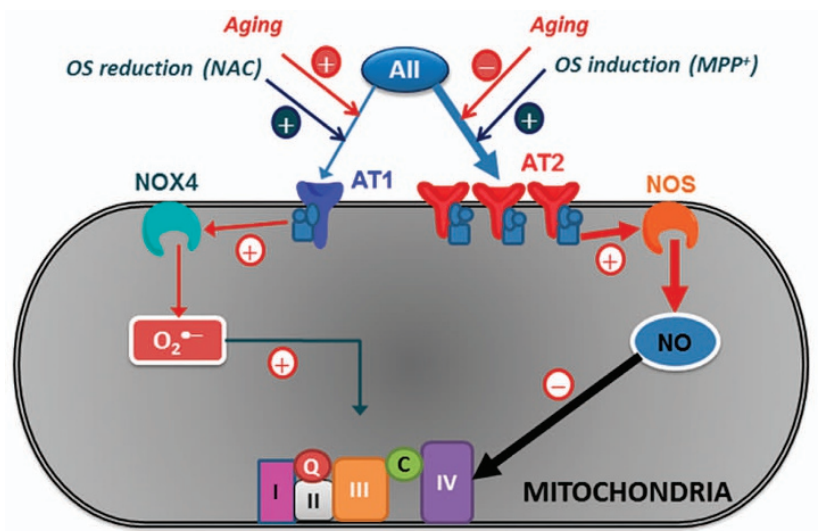

Figure 6 Model of the role that AT1 and AT2 receptors play in modulating oxidative phosphorylation in brain mitochondria. Activation of AT1 receptors in mitochondria regulates superoxide production, via Nox4, and increases respiration. Mitochondrial AT2 receptors are much more abundant and induce, via nitric oxide, a decrease in mitochondrial respiration, modulating oxidative phosphorylation without significant alteration in mitochondrial membrane potential, which indicates that the bioenergetic properties of the mitochondria are not affected. Mitochondrial AT2 receptor expression increased after treatment of cells with oxidative stress (OS) inducers (such as low doses of $\mathrm{MPP}^{+}$) and decreased with aging. Mitochondrial AT1 expression increased with aging and after treatment of cells with antioxidants (such as $\mathrm{N}$-acetyl-cysteine, NAC). At mitochondrial level, AT2 receptors may act as respiratory modulators and counteract low levels of OS, which may be particularly important in cells with an increase in levels of OS such as dopaminergic neurons. Aging induces altered expression of mitochondrial AT1 and AT2 receptors that may induce mitochondrial dysfunction, the main risk factor for neurodegeneration the involvement of $\mathrm{Nox} 4$ in mitochondrial $\mathrm{O}_{2}^{-}$production using small interfering RNA (siRNA) in isolated mitochondria from renal cells. ${ }^{16}$

In addition to the effect of mitochondrial AT1 receptors mentioned above, the activation of cell surface AT1 receptors and membrane-bound NADPH oxidase may also affect mitochondrial function. A number of studies have shown a ROS-mediated interaction (i.e. cross-talk signaling) between the membrane-bound NADPH oxidase complex and mitochondria, so that ROS generated by NADPH oxidase may act as a trigger to induce the opening of ATP-sensitive potassium channels (mitoKATP), which leads to generation of mitochondrial ROS. ${ }^{28}$ In cultures of dopaminergic neurons, we previously observed that inhibition of mitoKATP channels inhibits the All-induced increase in $\mathrm{O}_{2}^{-}$production. ${ }^{29}$ Mitochondrial AT1 and AT2 receptors may play a major role in maintaining the integrity of this essential organelle against extra-mitochondrial insults, at least in the early moments, as observed in the present experiments with isolated mitochondria. Consistent with this, an increase in cell levels of oxidative stress (i.e. treatment of dopaminergic cells with very low doses of $\mathrm{MPP}^{+}$) led to an increase in the expression of mitochondrial AT2 receptors. Interestingly, the present findings also show that aging modifies mitochondrial All receptor expression and the above-mentioned response to the increase in cell levels of oxidative stress. A number of previous studies have shown proinflammatory and pro-oxidative changes in the SN and different tissues of aged rats, and that age-related changes in RAS activity are involved in these changes and in the increased vulnerability of dopaminergic neurons with aging. ${ }^{30,31}$ Although an upregulation of mitochondrial AT2 receptors in response to the aging-related oxidative state may be expected, the present study shows an increase in mitochondrial AT1 receptor expression and a decrease in mitochondrial AT2 receptor expression in mitochondria isolated from old rats relative to those isolated from young rats. This may contribute to the increased vulnerability of these cells by inducing more mitochondrial oxidative stress and changes in respiratory efficiency, and play an important role in the development of aged-related neurodegenerative disorders such as PD. ${ }^{32,33}$

In summary (Figure 6), we conclude that functional AT1 and AT2 receptors exist in brain mitochondria, and particularly in dopaminergic neurons. Activation of mitochondrial AT1 induced superoxide production, via mitochondrial NOX4, and increased both mitochondrial respiration and ETS max $_{\text {. Mito- }}$ chondrial AT2 activation caused an NO-mediated reduction of mitochondrial respiration and ETS $_{\max }$, modulating oxidative phosphorylation without significant alteration in mitochondrial membrane potential, which indicates that the bioenergetic properties of the mitochondria are not affected. Mitochondrial AT2 receptors, which are clearly more abundant compared with mitochondrial AT1 in young rats, may counteract or modulate at the mitochondrial level the pro-oxidative effects of All stimulation of the cell membrane and mitochondrial AT1 receptors. AT2-mediated regulation of mitochondrial respiration may represent an unrecognized primary line of defence against oxidative stress, which may be particularly important in neurons with increased levels of oxidative stress such as dopaminergic neurons. 
An increase in mitochondrial AT1 and a decrease in mitochondrial AT2 expression in the nigral region of aged rats relative to young rats may play a major role in the mitochondrial dysfunction associated with normal aging, which is the major risk factor for the development of PD and other neurodegenerative diseases. Different types of drugs acting on the local RAS are currently used in vascular and renal diseases, and have been suggested as potential treatments for neurodegenerative diseases, including PD, based on their effects on surface All receptors. However, the functional effects of mitochondrial receptors, particularly AT2 receptors, must be taken into account in the design of new therapeutic strategies, and especially in diseases associated with excessive oxidative stress such as PD, but also diabetes, obesity and most of the cardiovascular diseases.

\begin{abstract}
Materials and Methods
Experimental design. Brain sections from adult male Sprague-Dawley rat containing SN, primary cultures from the nigral region and cultures of the dopaminergic neuron cell line MES 23.5 were used to investigate the presence of All receptors in mitochondria from dopaminergic neurons by immunofluorescence and electron microscopy. All experiments were carried out in accordance with Directive 2010/63/EU and Directive 86/609/CEE and were approved by the corresponding committee at the University of Santiago de Compostela. Animals were housed at constant room temperature $(\mathrm{RT})\left(21-22^{\circ} \mathrm{C}\right)$ and 12-h light/dark cycle. All surgery was performed under ketamine/xylazine anesthesia. The localization of angiotensinogen and angiotensin receptors in dopaminergic neurons isolated from rat SN was confirmed by LCM and RT-PCR. In addition, pure isolated mitochondria from the nigral region of rat were used to confirm the expression of All receptors in mitochondria using WB assay. Dopaminergic cells were also transfected with fluorescence-tagged AT1 and AT2 receptors, or treated with fluorescent All to investigate colocalization with mitochondrial markers.

Mitochondria isolated from the nigral region of rat in the VM and from AT1 and AT2 receptor knockout mice (Agtr1a C57BL/6 background and Agtr2 FVB background mutant mice) brain were used to investigate the ability of All receptors to modulate mitochondrial respiration, mitochondrial membrane potential and mitochondrial superoxide and nitric oxide (NO) production. For this purpose, mitochondria were incubated with different compounds to assess the effects of activation with either AT1 or AT2 on respiratory capacity and maintenance of membrane potential. Mitochondria were treated with All (1 nM) and pre-incubated with the AT2 antagonist PD123,319 $(2 \mu \mathrm{M})$ or the AT1 antagonist losartan $(3 \mu \mathrm{M})$, and/or the NADPH oxidase, NOX4, inhibitor thioridazine $(10 \mu \mathrm{M})$ or the NOS inhibitor L-NAME $(100 \mu \mathrm{M})$. The concentrations of the above-mentioned compounds were experimentally determined as the most appropriate for evaluating the studied effects. Mitochondrial oxidative stress was estimated measuring superoxide production (i.e. the most important reactive oxygen species, ROS, in mitochondria) using a sensitive luminescencebased assay. Finally, RT-PCR of nigral dopaminergic neurons isolated by LCM and WB assays of mitochondria isolated from young adult and aged male SpragueDawley rats (10 weeks and 20 months respectively) were used to investigate agerelated changes in dopaminergic neuron and mitochondrial All receptor expression.
\end{abstract}

Primary cultures from the nigral region and cultures of the MES 23.5 dopaminergic neurons. Ventral mesencephalic tissue was dissected from rat embryos of 14 days of gestation (E14). The tissue was incubated in $0.1 \%$ trypsin (Sigma, St. Louis, MO, USA), 0.05\% DNase (Sigma) and DMEM (Invitrogen, Paisley, Scotland, UK) for $20 \mathrm{~min}$ at $37^{\circ} \mathrm{C}$, and was then washed in DNase/DMEM and mechanically dissociated. The resulting cell suspension was centrifuged at $50 \times g$ for $5 \mathrm{~min}$, the supernatant was carefully removed and the pellet resuspended in $0.05 \%$ DNase/DMEM to the final volume required. The number of viable cells in the suspension was estimated with acridine orange/ethidium bromide. Cells were plated onto 35-mm culture dishes (Falcon; Becton Dickinson, Franklin Lakes, NJ, USA) previously coated with poly-L-lysine (100 $\mu \mathrm{g} / \mathrm{ml}$; Sigma) and laminin $(4 \mu \mathrm{g} / \mathrm{ml}$; Sigma). The cells were seeded at a density of $1.5 \times 10^{5}$ cells $/ \mathrm{cm}^{2}$ and maintained under control conditions (DMEM/HAMS F12/(1:1) containing $10 \%$ fetal bovine serum (FBS; Biochrom KG, Berlin, Germany)). The cell cultures were maintained in a humidified $\mathrm{CO}_{2}$ incubator $\left(5 \% \mathrm{CO}_{2} ; 37^{\circ} \mathrm{C}\right)$ for 8 days in vitro (DIV; see below); the entire culture medium was removed on day 2 and replaced with fresh culture medium.

Dopaminergic MES 23.5 cells, a gift from Dr. Wei-Dong Le (Baylor College of Medicine, Houston, TX, USA), were cultured in DMEM/F12 containing Sato's components growth medium supplemented with $2 \% \mathrm{FBS}, 100 \mathrm{units} / \mathrm{ml}$ penicillin and $100 \mu \mathrm{g} / \mathrm{ml}$ streptomycin at $37^{\circ} \mathrm{C}$ in a humidified $\mathrm{CO}_{2}$ incubator $\left(5 \% \mathrm{CO}_{2}, 95 \%\right.$ air). ${ }^{34}$ MES 23.5 cells were plated at a density of $0.5 \times 10^{5} / \mathrm{cm}^{2}$ onto $35-\mathrm{mm}$ plastic dishes with glass coverslips previously coated with poly-L-lysine (Sigma; $10 \mathrm{mg} / \mathrm{ml}$ ). To enhance differentiation, cells were stimulated by adding dibutyryl-cAMP (D0627, Sigma; $1 \mathrm{mM}$ ) to the supplemented growth medium.

Triple fluorescent labeling of cultures. Cultures grown on glass coverslips were incubated with the fluorescent probe Mitotracker Deep Red (MTDR; 20 nM; Molecular Probes, Waltham, MA, USA) for mitochondrial labeling. After mitochondrial labeling, cultures were fixed with $4 \%$ paraformaldehyde in Dulbecco's phosphate-buffered saline ( $\mathrm{pH} 7.4)$ for $20 \mathrm{~min}$, and were then processed for double immunofluorescence fluorescence. Cultures were incubated at $4{ }^{\circ} \mathrm{C}$ with a mouse monoclonal anti-TH (Sigma; $1: 30$ 000) antibody as dopaminergic marker and goat polyclonal anti-AT1 (sc-31181; 1 : 50; Santa Cruz Biotechnology) or rabbit polyclonal anti-AT2 receptor (sc-9040; 1 : 50; Santa Cruz Biotechnology) antibodies. The immunoreaction was visualized with the following fluorescent secondary antibodies: Alexa Fluor 405-conjugated donkey anti-mouse IgG (1:200; Abcam, Cambridge, England, UK) or biotinylated horse anti-goat IgG (BA9500, 1:50; Vector Labs, Burlingame, CA, USA) or biotinylated swine anti-rabbit IgG $(1: 200$; Dako, Barcelona, Spain) plus Alexa Fluor 488-conjugated Streptavidin (1:2500; Molecular Probes). Colocalization of markers was confirmed by confocal laser microscopy (TCS-SP2; Leica, Heidelberg, Germany) performing sequential scan to avoid any potential overlap. In all experiments, the control cultures, in which the primary antibody was omitted, were immunonegative for these markers.

Immuno electron microscopy for TH and AT1 and AT2 receptors. Adult male Sprague-Dawley rats were deeply anesthetized with a mixture of ketamine/xylazine and perfused with saline $(0.09 \% \mathrm{NaCl})$ followed by a solution of $4 \%$ paraformaldehyde and $0.1 \%$ glutaraldehyde in $0.1 \mathrm{M}$ phosphate buffer $\mathrm{pH} 7.4$ (PB). After perfusion, brains were quickly removed from the skull, immersed in the same fixative solution overnight at $4{ }^{\circ} \mathrm{C}$ and rinsed thoroughly in PB. Free-floating $40 \mu \mathrm{m}$ coronal sections were obtained on a vibratome and stored in PB until use. Sections containing the SN were transferred to citrate buffer pH 6.0 (Vector Laboratories; $\mathrm{H}-3300$ ) at RT for $5 \mathrm{~min}$ and then to citrate buffer at $80^{\circ} \mathrm{C}$ for $30 \mathrm{~min}$. Sections were rinsed consecutively in citrate buffer and phosphate-buffered saline (PBS) pH 7.4 at RT, prior to pre-treatment with $1 \%$ sodium borohydride in PBS for $15 \mathrm{~min}$. The sections were then rinsed several times in PBS, and transferred to a solution of $5 \%$ hydrogen peroxide in PBS for $30 \mathrm{~min}$, and rinsed several times in PBS. Sections were pre-incubated in 10\% normal serum containing $0.01 \%$ triton $\mathrm{X}-100$ in PBS for $1 \mathrm{~h}$ and then incubated for $72 \mathrm{~h}$ at $4{ }^{\circ} \mathrm{C}$ with the appropriate primary antibody: mouse monoclonal anti-TH (Sigma; 1: 10000 ), and AT1 goat polyclonal diluted $1: 100$ or AT2 rabbit polyclonal diluted 1:200, Santa Cruz Biotechnologies catalog number sc-31181 and sc-9040, respectively. Sections were rinsed thoroughly in PBS and incubated for $1 \mathrm{~h}$ with the appropriate secondary antibodies (biotinylated goat anti-rabbit or horse anti-goat; Vector Laboratories) diluted $1: 400$ in PBS containing $0.01 \%$ triton X-100, rinsed in PBS and incubated with an avidin-biotin-complex kit (Vector Laboratories; PK6100, diluted 1: 100) for $1 \mathrm{~h}$ at RT. Sections were rinsed multiple times in PBS, developed using 3,3'diaminobenzidine peroxidase kit (Vector SK4100) and rinsed in PBS.

Sections immunolabeled for AT1, AT2 or TH were rinsed in PB, transferred to a solution of $1 \%$ osmium tetroxide in $\mathrm{PB}$ for $1 \mathrm{~h}$ at $\mathrm{RT}$ and rinsed in $\mathrm{PB}$ prior to gradual dehydration in $50-70 \%$ ethanol. The sections were then transferred to a contrast solution containing $1 \%$ uranyl acetate in $70 \%$ ethanol for $1 \mathrm{~h}$ at RT. Sections were then rinsed in $70 \%$ ethanol to remove excess uranyl acetate and gradually dehydrated in $5 \mathrm{~min}$ baths of $70-100 \%$ ethanol. Sections were cleared in propylene oxide and gradually infiltrated with Epon resin by sequential immersion in a $2: 1$ mixture of propylene oxide and Epon resin (30 min), $1: 1$ mixture of propylene oxide and Epon resin (1 h), 1:2 mixture of propylene oxide and epon (1 h) and finally transferring the sections to Epon resin overnight at $4{ }^{\circ} \mathrm{C}$. The following day the sections were transferred to freshly prepared Epon resin for $1 \mathrm{~h}$ at RT, flat embedded and allowed to polymerize for a minimum of $72 \mathrm{~h}$ at $60^{\circ} \mathrm{C}$. After flat embedding was completed, the SN was clearly identified using a brightfield microscope and redissected for ultramicrotomy. Semithin (1- $\mu \mathrm{m}$-thick) and ultrathin (90-nm-thick) sections were cut using a Leica EM UC6 ultramicrotome (Leica Microsystems; Wetzlar, 
Germany). Ultrathin sections were placed on copper grids and observed and photographed using a Hitachi transmission electron microscopy (Hitachi, Tokyo, Japan) equipped with a Hamamatsu Orca digital camera (Hamamatsu, Hamamatsu, Japan).

Specificity of antibodies. The specificity of the antibodies used for WB and immunolabeling studies was established in previous studies: AT 1 sc- $31181^{35}$ and AT2 sc-9040. ${ }^{36,37}$ In addition, the specificity of the antibodies was confirmed in our laboratory by preadsortion with the corresponding synthetic peptide antigen ${ }^{38}$ In the present study, we also used WB analysis of lysates from HEK293 cells transfected with AT1 or AT2 tagged to fusion tail DDK (TA50011 from Origene, Rockville, MD, USA; DDK tag: DYKDDDDK). The specificity of the antibodies was confirmed by the presence of a predominant immunoreactive band in positively transfected lysates and the absence of this band in negative controls, which consisted of lysates transfected with empty vectors (Figure $1 \mathrm{~b}$ ).

Retrograde tracing, LCM and RT-PCR. Young adult (10-week-old, $n=3$ ) and aged (18-20-month-old, $n=4$ ) male Sprague-Dawley rats were used for LCM studies. Rats received three intrastriatal injections of red retrobeads $(0,5 \mu /$ injection; Lumafluor Inc., Durham, NC, USA) under ketamine/medetomidine anesthesia. The tracer was injected using a $10 \mu \mathrm{l}$ Hamilton syringe at a rate of $0.1 \mu \mathrm{l} / \mathrm{min}$. The stereotaxic coordinates for the injections were: bregma: $+1,-0.1$ or $-1.2 \mathrm{~mm}$; lateral: $\pm 3, \pm 3.7$ or $\pm 4.5 \mathrm{~mm}$; ventral: $5 \mathrm{~mm}$ from dura; tooth bar: 0 . Forty-eight hours after injection, rats were killed, brains were removed, snap-frozen in liquid nitrogen and stored at $-80^{\circ} \mathrm{C}$. Serial coronal sections $(20-\mu \mathrm{m}$-thick) including the VM were cut on a cryotome and mounted on RNase-free ultraviolet light-treated glass slides. Slides were immediately frozen at $-80^{\circ} \mathrm{C}$, and stored in sterile Falcon tubes containing silica gel. A series of $\mathrm{SN}$ sections were processed for immunofluorescence with an antibody against TH in order to verify the localization of red retrobeads within nigral dopaminergic neurons. LCM was performed using a PALM laser microbeam system (Zeiss, Jena, Germany) with a fluorescence option. Before LCM, frozen sections were allowed to equilibrate to increasing temperatures $\left(-20{ }^{\circ} \mathrm{C}, 4^{\circ} \mathrm{C}, \mathrm{RT}\right)$, fixed with ethanol $(70,96,100,100 \%)$ and dried at RT. Red retrobeads-labeled neurons were visualized ( $\times 40$ objective) within the SN, marked under fluorescence illumination and then cut and catapulted under brightfield microscopy. Neuronal cell pools were catapulted into an adhesive cap (Zeiss). For RNA extraction, trizol (Invitrogen) was added directly through the lid, the tube was shaken upside-down and then spun. Two thousand labeled dopaminergic neurons per animal were used for total RNA extraction according to the manufacturer's instructions. Homogenates of rat VM were used as a positive control.

Total RNA was reverse-transcribed to cDNA with nucleoside triphosphates containing deoxyribose, random primers and M-MLV reverse transcriptase (50U; Invitrogen). Real-time PCR was used to examine the relative levels of $\mathrm{TH}$, angiotensinogen, AT1 and AT2 mRNA. A real-time iCycler PCR platform (Bio Rad, Hercules, CA, USA) and IQ SYBR Green Supermix kit (Bio Rad) were used. $\beta$-Actin was used as a housekeeping gene and the data were evaluated by the delta-delta $\mathrm{Ct}$ method $(2-\Delta \Delta \mathrm{Ct})$, where $\mathrm{Ct}$ is the cycle threshold. Finally, the PCR products were loaded on a $2 \%$ agarose gel with SYBR Safe stain (Invitrogen) and separated by electrophoresis. Primer sequences were as follows: for AT1, forward $5^{\prime}$ TTCAACCTCTACGCCAGTGTG-3', reverse 5'-GCCAAGCCAGCCATCAGC-3'; for AT2, forward 5'-AACATCTGCTGAAGACCAATAG-3', reverse 5'-AGAAGGTCAGAA CATGGAAGG-3'; for angiotensinogen, forward $5^{\prime}$-GAGTGAGGCAAGAGGTGTA-3', reverse $5^{\prime}$-TCCAACGATCCAAGGTAGAA-3'; for TH, forward $5^{\prime}$-GGCTTCTCTGAC CAGGTGTATCG-3', reverse 5'-GCAATCTCTTCCGCTGTGTATTCC-3'; and for $\beta$-actin, forward 5'-TCGTGCGTGACATTAAAGAG-3', reverse 5'-TGCCACAGGATT CCATACC- $3^{\prime}$

Transient transfection of All receptors and Alexa Fluor 488conjugated All treatment of dopaminergic cell line MES 23.5. The dopaminergic neuron cell line MES 23.5 was cultured in DMEM/F12 containing Sato's components growth medium supplemented with $2 \%$ FBS, 100 units $/ \mathrm{ml}$ penicillin and $100 \mu \mathrm{g} / \mathrm{ml}$ streptomycin at $37^{\circ} \mathrm{C}$ in a humidified $\mathrm{CO}_{2}$ incubator $(5 \%$ $\mathrm{CO}_{2}, 95 \%$ air). MES 23.5 cells were plated at a density of $0.3 \times 10^{5} / \mathrm{cm}^{2}$ onto $35-\mathrm{mm}$ plastic dishes with glass coverslips previously coated with poly-L-lysine (Sigma; $10 \mathrm{mg} / \mathrm{ml}$ ). In the first series of experiments, cells were treated with MitoTracker Deep Red $633(20 \mathrm{nM}, 15 \mathrm{~min})$ to label mitochondria, and transiently transfected with $1 \mu \mathrm{g}$ of AT1 cDNA (AT1/EGFP-N3) and $3 \mu \mathrm{g}$ of AT2 cDNA (AT2/YFP-N1) for $24 \mathrm{~h}$ using Lipofectamine LTX transfection reagent (Invitrogen). To study the effects of pro-oxidative and antioxidative conditions, some cultures of cells transfected with AT2-yellow fluorescent protein (YFP) receptor were treated with a low dose of the dopaminergic neurotoxin MPP ${ }^{+}$iodide $(10 \mu \mathrm{M}$; Sigma) for $24 \mathrm{~h}(n=4)$, and cells transfected with AT1-enhanced green fluorescent protein (EGFP) receptor were treated with the antioxidant $\mathrm{N}$-acetyl-L-cysteine $(0.5 \mathrm{mM}$; Sigma) for $24 \mathrm{~h}(n=3)$. The effect of these treatments on expression of mitochondrial AT1 and AT2 receptors were observed by confocal microscopy and confirmed by WB of isolated mitochondria from MES 23.5 cells using antibodies against GFP (Life Technologies, Waltham, MA, USA; G10362; $1: 800$ ), AT1 and AT2 (Santa Cruz Biotechnology; sc31181 and Sc-9040) and VDAC/porin (Sigma; V2139; 1:1,000). The purity of isolated mitochondria from MES 23.5 cells was previously demonstrated by the absence of other cellular compartment markers (see below). In order to minimize the toxicity of AT1 receptor overexpression, cells transfected with AT1-EGFP required the simultaneous treatment with the AT1 receptor antagonist losartan $(3 \mu \mathrm{M})$, which was essential to maintain cell survival.

In a second series of experiments we investigated the presence of All in mitochondria. MES 23.5 cells were treated with the mitochondrial marker MitoTracker Deep Red 633 (20 nM, $15 \mathrm{~min})$ and Alexa Fluor 488-conjugated All $(500 \mathrm{nM}$; Molecular Probes). Cells were fixed in $4 \%$ paraformaldehyde for different lengths of time between 30 min and $8 \mathrm{~h}$, and fluorescence images were acquired with a Leica SP5 confocal laser scanning microscope.

Mitochondrial isolation. Mitochondria from nigral region and whole brain of rat, and from AT1a and AT2 receptor knockout mice (Agtr1a and Agtr2 mutant mice) brain, were isolated and purified according to the protocol described by Sims and Anderson ${ }^{14}$ with some modifications. This protocol combines differential centrifugation and discontinuous Percoll density gradient centrifugation to isolate pure mitochondria with scarce contamination by synaptosomes and myelin. Rat nigral region was removed and washed in cold isolation buffer $(0.32 \mathrm{M}$ sucrose, $1 \mathrm{mM}$ and $10 \mathrm{mM}$ TRIS; $\mathrm{pH}$ 7.4). The tissue was cut into small pieces, transferred to a Dounce homogenizer with $12 \%$ Percoll solution, and then homogenized on ice using a loose fitting and tight fitting glass pestles. The homogenate was slowly layered on a previously prepared discontinuous Percoll gradient consisting of $26 \%$ Percoll layered over $40 \%$ Percoll, and centrifuged using a fixed-angle rotor at $30700 \times \mathrm{g}$ for $5 \mathrm{~min}$ at $4{ }^{\circ} \mathrm{C}$. This produced three separate bands, and the enriched mitochondrial fraction, which appeared at the interface between the 26 and $40 \%$ Percoll layers, was carefully removed with a glass Pasteur pipette. The mitochondrial fraction was diluted by adding isolation buffer, and was again centrifuged at $16700 \times \mathrm{g}$ for $10 \mathrm{~min}$ at $4{ }^{\circ} \mathrm{C}$. This yielded a mitochondrial pellet, which was gently resuspended in the residual supernatant. Finally, the pellet was resuspended in isolation buffer and centrifuged at $7300 \times \mathrm{g}$ for $10 \mathrm{~min}$ at $4{ }^{\circ} \mathrm{C}$, yielding a pellet of pure mitochondria that was used for WB and functional assays. For WB confirmation of the presence of transfected angiotensin receptors in mitochondria of the dopaminergic cell line MES 23.5, mitochondria were isolated with a Mitochondria Isolation kit (Mitosciences; ab110170) for cultured cells.

Western blot of mitochondria from rat nigral region and MES 23.5 dopaminergic neurons. Protein concentration in the isolated mitochondria from the nigral region $(n=4)$ and whole homogenate $(n=4)$ were quantified using the Pierce BCA Protein Assay Kit (Thermo Scientific, Fremont, CA, USA). Equal amounts of protein were separated on a $5-10 \%$ bis-tris polyacrylamide gel, and transferred to nitrocellulose membranes. Membranes were incubated overnight with primary antibodies against VDAC/porin (V2139, 1:1000) and $\alpha$-Tubulin (T5168, 1:50 000) from Sigma, HDAC2 (sc-56685, 1:200), AT1 (sc-31181, $1: 200$ ) and AT2 (sc-9040, $1: 200)$ from Santa Cruz Biotechnology and rabbit monoclonal antibody to Nox4 (ab133303, $1: 800$ ) from Abcam. The following HRP-conjugated secondary antibodies were used: Protein A (1:5000) (NA9120V; GE Healthcare), chicken anti-mouse lgG-HRP (sc-2954, $1: 2500$ ) and donkey antigoat IgG-HRP (sc-2020, $1: 2500$ ) from Santa Cruz Biotechnology. Immunoreactive bands were detected with an Immun-Star HRP Chemiluminescent Kit (170-5044; Bio Rad) and visualized with a chemiluminescence detection system (Molecular Imager ChemiDoc XRS System; Bio Rad). Specificity of the antibodies was confirmed as indicated above.

Detection of All in purified isolated mitochondria by HPLC and specific All EIA. For the separation of angiotensin peptides, mitochondria were isolated as described above and stored at $-20^{\circ} \mathrm{C}$. The mitochondrial pellet was reconstituted in $100 \mu \mathrm{l}$ of Milli-Q water and heated at $96{ }^{\circ} \mathrm{C}$ for $15 \mathrm{~min}$. The mitochondrial fraction was acidified with heptafluorobutyric acid (HFBA) to a final concentration of $0.1 \%$, sonicated and centrifuged at $20000 \times g$ for $20 \mathrm{~min}$ at $4{ }^{\circ} \mathrm{C}$. The supernatants were applied to a Sep Pak columns (Oasis HLB 1cc, 
WAT094225) pre-conditioned with $1 \mathrm{ml}$ of methanol and deionized water. The loaded Sep Pak columns were washed with $1 \mathrm{ml}$ of $0.1 \%$ HFBA in deionized water All and its fragments were eluted with $1 \mathrm{ml}$ of methanol in $0.1 \%$ of HFBA, and the eluent dried in a vacuum concentrator (Savant ISS110). Dried samples $(n=5)$ were resuspended in $60 \mu \mathrm{l}$ of a solution of $17 \%$ acetonitrile in $4 \mathrm{mM}$ TEAF with $30 \mathrm{mM}$ formic acid and injected ( $20 \mu \mathrm{l} /$ injection) into the HPLC system. Peptides were separated at $35^{\circ} \mathrm{C}$ and a flow rate of $1 \mathrm{ml} / \mathrm{min}$ with an acetonitrile gradient on a reverse phase C18 column (Waters Symmetry300C18; $150 \times 3.9 \mathrm{~mm}, 5 \mu \mathrm{m}$ particle size; Waters, Barcelona, Spain). For the acetonitrile gradient, solution A was made with $30 \mathrm{mM}$ formic acid in $4 \mathrm{mM}$ TEAF. Solution B consisted of $90 \%$ acetonitrile in $4 \mathrm{mM}$ TEAF with $30 \mathrm{mM}$ formic acid. The linear gradient used was from 11 to $50 \%$ of $B$ in 20 min. Eluate from the column was monitored at a wavelength of $220 \mathrm{~nm}$ in an UV-Vis detector (SPD-20AV; Shimadzu, Columbia, MD, USA). All fractions were collected with a fraction collector (FRC-10A; Shimadzu), dried in a vacuum concentrator and stored at $-80^{\circ} \mathrm{C}$ until analysis of All content by EIA kit (A05880; SpiBio, Frankston, TX, USA) following the manufacturer's specifications.

High-resolution respirometry. Mitochondrial respiration was measured by high-resolution respirometry at $37^{\circ} \mathrm{C}$ using an Oxygraph-2k respirometer (OROBOROS Instruments, Innsbruck, Austria). Aliquots of $80-120 \mu \mathrm{g}$ of isolated mitochondria were incubated with AT1 or AT2 receptor antagonists $(3 \mu \mathrm{M}$ losartan or $2 \mu \mathrm{M}$ PD123,319, respectively; $n=3-8)$ for $3 \mathrm{~min}$, and treated with $1 \mathrm{nM}$ All for 5 min. To know the effects of NO and NOX4, the NOS inhibitor L-NAME or the NOX4 inhibitor thioridazine were added to mitochondria (see below) with or without corresponding treatment in respiration buffer (125 mM KCl, $5 \mathrm{mM}$ HEPES, $3 \mathrm{mM}$ $\mathrm{MgCl}_{2}, 2 \mathrm{mM} \mathrm{KH} 2 \mathrm{PO}_{4}$ and $0.5 \mathrm{mM}$ EGTA; $\mathrm{pH}$ 7.4) using a substrate-uncouplerinhibitor titration protocol (SUIT), as previously described. ${ }^{39}$ The substrates used to fuel the ETS were $2 \mathrm{mM}$ malate $+5 \mathrm{mM}$ pyruvate (MP) for evaluating complex respiration. The OXPHOS capacity, which is similar to State 3 , was measured in the presence of substrates with saturating ADP $(2.5 \mathrm{mM})$. The maximum ETS capacity (ETS $_{\text {max }}$; i.e. non-coupled respiration) was evaluated using carbonyl cyanide-4(trifluoromethoxy)phenylhydrazone (FCCP; $0.5-1.5 \mu \mathrm{M}$ ) as the uncoupler. The integrity and function of our mitochondrial preparations were confirmed by addition of exogenous cytochrome $c$, which did not enhance mitochondrial respiration, indicating nonsignificant damage to the outer mitochondrial membrane during isolation procedures (data not shown). Respiration rates $\left(\mathrm{O}_{2}\right.$ flux) were calculated as the negative time derivative of oxygen concentration, and Datlab software (OROBOROS Instruments) was used for both data acquisition and analysis. Respiration rates were normalized to the amount of mitochondrial protein to allow comparison between groups without the influence of differences in the amount of mitochondria loaded into the chamber.

Mitochondrial membrane potential. Membrane potential of mitochondria isolated from rat brain was measured using the sensitive lipophilic cationic

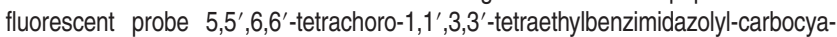
nine iodide (JC-1; Sigma) as previously described. ${ }^{40}$ Isolated mitochondria were treated with different compounds and respiratory substrates for $10 \mathrm{~min}$ at $37^{\circ} \mathrm{C}$. Mitochondria were treated with All $(1 \mathrm{nM})$ and the AT2 antagonist PD123,319 $(2 \mu \mathrm{M})$ or the AT1 antagonist losartan $(3 \mu \mathrm{M})$ to investigate the effect of mitochondrial AT1 and AT2 receptors on mitochondrial membrane potential. Samples $(n=5)$ were then stained with $\mathrm{JC}-1(0.2 \mu \mathrm{g} / \mathrm{ml}$; previously incubated on ice for $20 \mathrm{~min}$ ) and the red fluorescence of dye agglomerates was measured for $15 \mathrm{~min}$ (excitation/emission wavelength $=490 / 590 \mathrm{~nm}$ ) in a fluorescence plate reader (Tecan; Infinite M200). Valinomycin $(0.5 \mu \mathrm{g} / \mathrm{ml})$, a potassium ionophore, was used as a control to permeabilize the mitochondrial membrane for $\mathrm{K}^{+}$ions, dissipating the mitochondrial electrochemical potential and preventing JC-1 aggregation.

Superoxide production and nitric oxide production assays. Pure mitochondrial fractions obtained with Percoll gradients were directly assayed for superoxide $\left(\mathrm{O}_{2}^{-}\right)$production using lucigenin-enhanced chemiluminescence as follows. ${ }^{16,17}$ Lucigenin is an acridylium dinitrate compound that on reducing and interacting with superoxide anions emits light that is quantified to measure the production of $\mathrm{O}_{2}^{-}$. An aliquot of $30 \mu \mathrm{g}$ mitochondria $(n=4-8)$ was incubated for $10 \mathrm{~min}$ at $37^{\circ} \mathrm{C}$ with $1 \mathrm{nM}$ All and pre-incubated with the AT2 receptor antagonist PD123,319 $(2 \mu \mathrm{M})$ in $50 \mathrm{mM}$ phosphate buffer, pH 7.0 containing $1 \mathrm{mM}$ EGTA and $150 \mathrm{mM}$ sucrose. Five micromolar lucigenin and $100 \mu \mathrm{M}$ NADPH were then added to start the reaction. Photon emission, in terms of relative chemiluminescence units (RLU), was measured every $20 \mathrm{~s}$ for $10 \mathrm{~min}$ using a luminometer plate reader
(Tecan; Infinite M200; Männedorf, Switzerland). There was no measurable activity in the absence of NADPH. The Nox4 inhibitor thioridazine $(10 \mu \mathrm{M})$ was used to determine the amount of $\mathrm{O}_{2}^{-}$produced by this mitochondrial member of the Nox/ NADPH oxidase family. The role of Nox4 in All-induced superoxide production was confirmed with a second Nox4 inhibitor (diphenyleneidonium, DPI). However, it has been suggested that DPI may also affect NOS. ${ }^{41,42}$ Mitochondrial NOS is related to the mitochondrial AT2 receptor effects and inhibition with DPI may be not adequate for the present study, particularly for respirometry. Therefore, we only included the results observed with thioridazine.

Mitochondrial nitric oxide (NO) production was measured with an NO fluorometric assay kit (Biovision, Milpitas, CA, USA). Because of its short half-life, direct measurement of $\mathrm{NO}$ is difficult; thus the total concentrations of nitrate and nitrite have been used as useful indicators of NO production. An aliquot of $30 \mu \mathrm{g}$ of mitochondria $(n=5-8)$ was incubated for 10 min with $1 \mathrm{nM}$ All and the AT1 antagonist losartan $(3 \mu \mathrm{M})$ to investigate the effect of mitochondrial AT2 receptors activation, and the effect of the NOS inhibitor L-NAME $(100 \mu \mathrm{M})$. Nitrate was then converted to nitrite by the nitrate reductase enzyme, and the total nitrite concentration was measured as nitrite reacted with the fluorescent probe DAN (2,3-diaminonaphthalene), which is proportional to the total nitric oxide production.

LDH and COX activities. LDH is a cytosolic enzyme predominantly associated with contaminating synaptosomes; thus, it was used to assess the purity of the mitochondrial fraction. LDH activity was measured in $2.5 \mu \mathrm{g}$ isolated mitochondria and whole homogenate protein $(n=6)$, according to the manufacturer's specifications (Roche 04744926001). The $\mathrm{micromol} / \mathrm{min} / \mathrm{mg}$ was calculated using LDH enzyme (Sigma, L-3916) as a reference standard.

The COX activity was used to normalize the data obtained from AT1 and AT2 receptor expression performed by WB in aged rats, as this provides the most accurate estimation of mitochondrial content. COX activity was measured in isolated mitochondria using a commercial kit according to the manufacturer's specifications (KC310100; Biochain, Newark, CA, USA). COX activity was measured in a 96-well plate by detecting the decrease in absorbance of ferrocytochrome $c$ at $550 \mathrm{~nm}$, as it is oxidized to ferricytochrome $c$ by COX. Ten micrograms of isolated mitochondria protein with $0.1 \%$ of DTT were assayed adding ferrocytochrome $c$ substrate and reading the absorption at $550 \mathrm{~nm}$ immediately for $55 \mathrm{~s}$. The COX activity (unit/ml) of each sample was calculated using the molar absorbance coefficient 21.84 for ferrocytochrome $c$ and ferricytochrome $c$ at $550 \mathrm{~nm}$

Statistical analysis. All data were obtained from at least three independent experiments and were expressed as mean values \pm S.E.M. Two-group comparisons were analyzed by a Student's $t$-test and multiple comparisons were analyzed by one-way ANOVA followed by a post hoc Bonferroni test. The normality of populations and homogeneity of variances were tested before each ANOVA. Differences were considered significant at $P<0.05$. Statistical analyses were carried out with SigmaStat 3.0 (Jandel Scientific, San Rafael, CA, USA).

\section{Conflict of Interest}

The authors declare no conflict of interest.

Acknowledgements. We thank Dr. Wei-Dong Le for providing the MES 23.5 dopaminergic cell line. We thank Pilar Aldrey, Iria Novoa, Begoña Gonzalez and Jose Trillo for their technical assistance. Grant sponsors of this work are Spanish Ministry of Economy and Competitiveness (BFU2012-37087), Spanish Ministry of Health (RD12/0019/0020 and CIBERNED), Galician Government (XUGA) and FEDER (Regional European Development Fund).

\section{Author contributions}

$\mathrm{RV}$ and MAC-B performed isolations of mitochondria, western blot, enzymatic activity determinations and other biochemical experiments. RV, JI-G and RS-O performed mitochondrial respirometry experiments. EP-C and MM-F performed electron microscopy experiments. RV, MAC-B and JLL performed cell cultures, confocal microscopy and immunohistochemical studies. RF prepared expression vectors with AT1 and AT2 receptor CDNA, and RV and BV-C performed transfections. PG-G and MAC-B performed in vivo studies, stereotactic injections of tracers, laser captured microdissection and RT-PCR. DH collaborated in the AT2 knockout mice studies. JLL- 
G and RV conceived and supervised the whole study and wrote the manuscript. All authors edited the manuscript.

1. Chabrashvili T, Kitiyakara C, Blau J, Karber A, Aslam S, Welch WJ et al. Effect of Ang II type 1 and 2 receptors on oxidative stress, renal $\mathrm{NAD}(\mathrm{P}) \mathrm{H}$ oxidase, and $\mathrm{SOD}$ expression. Am J Physiol Regu Integr Comp Physiol 2003; 285: R117-R124.

2. Re RN. Tissue renin angiotensin systems. Med Clin North Am 2004; 88: 19-38.

3. Labandeira-García JL, Garrido-Gil P, Rodriguez-Pallares J, Valenzuela R, Borrajo A, Rodríguez-Perez Al. Brain renin-angiotensin system and dopaminergic cell vulnerability. Front Neuroanat 2014; 8: 67.

4. Wright JW, Harding JW. The brain renin-angiotensin system: a diversity of functions and implications for CNS diseases. Pflugers Arch 2013; 465: 133-151.

5. Garrido-Gil P, Valenzuela R, Villar-Cheda B, Lanciego JL, Labandeira-Garcia JL. Expression of angiotensinogen and receptors for angiotensin and prorenin in the monkey and human substantia nigra: an intracellular reninangiotensin system in the nigra. Brain Struct Funct 2013; 218: 373-388

6. Labandeira-Garcia JL, Rodriguez-Pallares J, Dominguez-Meijide A, Valenzuela $R$, Villar-Cheda B, Rodríguez-Perez Al. Dopamine-angiotensin interactions in the basal ganglia and their relevance for Parkinson's disease. Mov Disord 2013; 28: 1337-1342.

7. Rodriguez-Pallares J, Rey P, Parga JA, Muñoz A, Guerra MJ, Labandeira-Garcia JL. Brain angiotensin enhances dopaminergic cell death via microglial activation and NADPHderived ROS. Neurobiol Dis 2008; 31: 58-73.

8. Grammatopoulos TN, Jones SM, Ahmadi FA, Hoover BR, Snell LD, Skoch J et al. Angiotensin type 1 receptor antagonist losartan, reduces MPTP-induced degeneration of dopaminergic neurons in substantia nigra. Mol Neurodegener 2007; 2: 1.

9. Rey P, Lopez-Real A, Sanchez-Iglesias S, Muñoz A, Soto-Otero R, Labandeira-Garcia JL. Angiotensin type-1-receptor antagonists reduce 6-hydroxydopamine toxicity for dopaminergic neurons. Neurobiol Aging 2007; 28: 555-567.

10. Lavoie JL, Cassell MD, Gross KW, Sigmund CD. Localization of renin expressing cells in the brain, by use of a REN-eGFP transgenic model. Physiol Genomics 2004; 16: 240-246.

11. Re RN. Intracellular renin and the nature of intracrine enzymes. Hypertension 2003; 42 117-122.

12. Baker KM, Chernin MI, Schreiber T, Sanghi S, Haiderzaidi S, Booz GW et al. Evidence of a novel intracrine mechanism in angiotensin II-induced cardiac hypertrophy. Regul Pept 2004; 120: $5-13$.

13. Valenzuela R, Barroso-Chinea P, Muñoz A, Joglar B, Villar-Cheda B, Lanciego JL et al. Location of prorenin receptors in primate substantia nigra: effects on dopaminergic cell death. J Neuropathol Exp Neurol 2010; 69: 1130-1142.

14. Sims NR, Anderson MF. Isolation of mitochondria from rat brain using Percoll density gradient centrifugation. Nat Protoc 2008; 3: 1228-1239.

15. Picard M, Ritchie D, Wright KJ, Romestaing C, Thomas MM, Rowan SL et al. Mitochondrial functional impairment with aging is exaggerated in isolated mitochondria compared to permeabilized myofibers. Aging Cell 2010; 9: 1032-1046.

16. Block K, Gorin Y, Abboud HE. Subcellular localization of Nox4 and regulation in diabetes. Proc Natl Acad Sci USA 2009; 106: 14385-14390.

17. Gorin Y, Ricono JM, Kim NH, Bhandari B, Choudhury GG, Abboud HE. Nox4 mediates angiotensin II-induced activation of Akt/protein kinase B in mesangial cells. Am J Physiol Renal Physiol 2003; 285: F219-F229.

18. Serrander L, Cartier L, Bedard K, Banfi B, Lardy B, Plastre $O$ et al. NOX4 activity is determined by mRNA levels and reveals a unique pattern of ROS generation. Biochem $\mathrm{J}$ 2007; 406: 105-114

19. Swerdlow RH. The neurodegenerative mitochondriopathies. J Alzheimers Dis 2009; 17: 737-751.

20. Federico A, Cardaioli E, Da Pozzo P, Formichi P, Gallus GN, Radi E. Mitochondria, oxidative stress and neurodegeneration. J Neurol Sci 2012; 322: 254-262.

21. Schapira AH. Mitochondria in the aetiology and pathogenesis of Parkinson's disease. Lancet Neurol 2008; 7: 97-109.

22. Brookes PS, Levonen AL, Shiva S, Sarti P, Darley-Usmar VM. Mitochondria: regulators of signal transduction by reactive oxygen and nitrogen species. Free Radic Biol Med 2002; 33: 755-764.

23. Beltrán B, Mathur A, Duchen MR, Erusalimsky JD, Moncada S. The effect of nitric oxide on cell respiration: a key to understanding its role in cell survival or death. Proc Natl Acad Sci USA 2000; 97: 14602-14607.

24. Paxinou E, Weisse M, Chen Q, Souza JM, Hertkorn C, Selak M et al. Dynamic regulation of metabolism and respiration by endogenously produced nitric oxide protects against oxidative stress. Proc Natl Acad Sci USA 2001; 98: 11575-11580.

25. Joglar B, Rodriguez-Pallares J, Rodríguez-Perez Al, Rey P, Guerra MJ, Labandeira-Garcia $\mathrm{JL}$. The inflammatory response in the MPTP model of Parkinson's disease is mediated by brain angiotensin: relevance to progression of the disease. J Neurochem 2009; 109: $656-669$.
26. Ago T, Matsushima S, Kuroda J, Zablocki D, Kitazono T, Sadoshima J. The NADPH oxidase Nox4 and aging in the heart. Aging 2010; 2: 1012-1016.

27. Kuroda J, Nakagawa K, Yamasaki T, Nakamura K, Takeya R, Kuribayashi F et al. The superoxide-producing $\mathrm{NAD}(\mathrm{P}) \mathrm{H}$ oxidase Nox4 in the nucleus of human vascular endothelial cells. Genes Cells 2005; 10: 1139-1151.

28. Zhang DX, Gutterman DD. Mitochondrial reactive oxygen species-mediated signaling in endothelial cells. Am J Physiol Heart Circ Physiol 2007; 292: H2023-H2031.

29. Rodriguez-Pallares J, Parga JA, Joglar B, Guerra MJ, Labandeira-Garcia JL. Mitochondrial ATP-sensitive potassium channels enhance angiotensin-induced oxidative damage and dopaminergic neuron degeneration. Relevance for aging-associated susceptibility to Parkinson's disease. Age 2012; 34: 863-880.

30. Villar-Cheda B, Valenzuela R, Rodriguez-Perez Al, Guerra MJ, Labandeira-Garcia JL. Aging-related changes in the nigral angiotensin system enhances proinflammatory and prooxidative markers and 6-OHDA-induced dopaminergic degeneration. Neurobiol Aging 2012; 33: 204-211.

31. Villar-Cheda B, Dominguez-Meijide A, Valenzuela R, Granado N, Moratalla R, LabandeiraGarcia JL. Aging-related dysregulation of dopamine and angiotensin receptor interaction. Neurobiol Aging 2014; 35: 1726-1738.

32. Mandemakers W, Morais VA, De Strooper B. A cell biological perspective on mitochondrial dysfunction in Parkinson disease and other neurodegenerative diseases. J Cell Sci 2007; 120: $1707-1716$

33. Jellinger KA. Recent advances in our understanding of neurodegeneration. J Neural Transm 2009: 116: 1111-1162.

34. Crawford GD, Le WD, Smith RG, Xie WJ, Stefani E, Appel SH. A novel N18TG2 x mesencephalon cell hybrid expresses properties that suggest a dopaminergic cell line of substantia nigra origin. J Neurosci 1992; 12: 3392-3398.

35. Giles ME, Fernley RT, Nakamura Y, Moeller I, Aldred GP, Ferraro T et al. Characterization of a specific antibody to the rat angiotensin II AT1 receptor. J Histochem Cytochem 1999; 47: 507-516.

36. Ruiz-Ortega M, Esteban V, Suzuki $\mathrm{Y}$, Ruperez M, Mezzano S, Ardiles L et al. Renal expression of angiotensin type 2 (AT2) receptors during kidney damage . Kidney Int 2003; 64: S21-S26.

37. Obermüller N, Gentili M, Gauer S, Gretz N, Weigel M, Geiger H et al. Immunohistochemical and $\mathrm{mRNA}$ localization of the angiotensin II receptor subtype 2 (AT2; in follicular granulosa cells of the rat ovary. J Histochem Cytochem 2004; 52: 545-548.

38. Rodriguez-Perez Al, Valenzuela R, Villar-Cheda B, Guerra MJ, Lanciego JL, LabandeiraGarcia JL. Estrogen and angiotensin interaction in the substantia nigra. Relevance to postmenopausal Parkinson's disease. Exp Neurol 2010; 224: 517-526.

39. Iglesias-Gonzalez J, Sanchez-Iglesias S, Mendez-Alvarez E, Rose S, Hikima A, Jenner P et al. Differential toxicity of 6-hydroxydopamine in SH-SY5Y human neuroblastoma cells and rat brain mitochondria: protective role of catalase and superoxide dismutase. Neurochem Res 2012; 37: 2150-2160.

40. Sareen D, Ginkel PR, Takach J, Mohiuddin A, Darjatmoko SR, Albert DM et al. Mitochondria as the primary target of resveratrol-induced apoptosis in human retinoblastoma cells. Invest. Ophthalmol Vis Sci 2006; 47: 3708-1716.

41. Lassegue $B$, Clempus RE. Vascular NAD(P)H oxidases: specific features, expression, and regulation. Am J Physiol Regul Integr Comp Physiol 2003; 285: R277-R297.

42. Wang $\mathrm{YX}$, Poon $\mathrm{Cl}$, Poon KS, Pang CC. Inhibitory actions of diphenyleneiodonium on endothelium-dependent vasodilatations in vitro and in vivo. Br J Pharmacol 1993; 110 1232-1238.

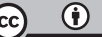
published by Nature Publishing Group. This work is licensed under a Creative Commons Attribution 4.0 International License. The images or other third party material in this article are included in the article's Creative Commons license, unless indicated otherwise in the credit line; if the material is not included under the Creative Commons license, users will need to obtain permission from the license holder to reproduce the material. To view a copy of this license, visit http://creativecommons.org/licenses/by/4.0/

(C) The Author(s) 2016 